\title{
Publishing Sensor Observations into Geospatial Information Infrastructures: A Use Case in Fire Danger Assessment
}

\author{
Laura Díaz1,2, Arne Bröring2,3, Daniel Mclnerney4, Giorgio Libertá4, Theodor Foerster² \\ ${ }^{1}$ Institute of New Imaging Technologies (SP) \\ ${ }^{2}$ Institute for Geoinformatics, University of Muenster (DE) \\ ${ }^{3} 52^{\circ}$ North Initiative for Geospatial Open Source Software, Muenster (DE) \\ ${ }^{4}$ Institute for Environment and Sustainability, European Commission, Joint Research Centre (IT)
}

laura.diaz@uji.es, arne.broering@wwu.de,daniel.mc-inerney@jrc.ec.europa.eu, giorgio.liberta@jrc.ec.europa.eu, theodor.foerster@wwu.de

\begin{abstract}
To improve environmental monitoring, the availability of great coverage of spatiotemporal data in an interoperable way is crucial for its integration into environmental models, for example, to compute fire danger models. To produce up-to-date and accurate results those models need the availability of data with high temporal and spatial resolution. Thus, it is promising to consider the increasing number of in-situ sensors providing observations of our environment in real-time. Today, interoperable access to such spatio-temporal data is achieved by Geospatial Information Infrastructures (GIIs). From a technical point of view GIIs provide this data through standards-based Web service interfaces. While those Web service interfaces already enable the interoperable discovery and retrieval of sensor observations, the functionality to publish sensor observations is still an arduous task. Hence, in this paper, we present an approach to improve the registration of sensors and the publication of their observations via standards-based Web service interfaces. We evaluate our approach by extending a standards-based GII and by applying the developed approach to the example of integrating in-situ weather observations into the European Forest Fire Information System for assessing fire danger in Spain.
\end{abstract}

Keywords: Geospatial Information Infrastructures; Spatial Data Infrastructures; Sensors; Sensor

Observation Service, OGC, Environmental Models; Fire Danger 


\section{Software availability}

Name of software: GEOSS Service Factory

Developers: Geospatial Technologies Research Group (Geotec), Universitat Jaume I of Castellón Contact information: Av Vicent Sos Baynat, s/n, Universitat Jaume I, 12071 Castellón, Spain

Software required: Servlet container such as Apache Tomcat and a Internet browser to invoke it

(Firefox, Internet Explorer, Chrome, etc.)

Program language: Java

Availability and cost: Users can access binaries and source code freely

Documentation and code available at:

http://www.geotec.uji.es/?page id=649

Name of software: Geotec Meteorological SOS based on $\mathbf{5 2}^{\circ}$ North SOS

Developers: $52^{\circ}$ North GmbH \& Geospatial Technologies Research Group (Geotec),

Contact information: Av Vicent Sos Baynat, s/n, Universitat Jaume I, 12071 Castellón, Spain

Software required: Spatial Database, Servlet container such as Apache Tomcat and an Internet

browser to invoke it (Firefox, Internet Explorer, Chrome, etc.)

Program language: Java

Availability and cost: Users can access binaries and source code freely

Documentation and code available at:

http://52north.org/sos

Geotec SOS instance available at:

http://geo3.dlsi.uji.es:8080/SOSMeteo/sos

Name of software: SID Interpreter

Developers: $52^{\circ} \mathrm{North} \mathrm{GmbH}$

Contact information: Arne Bröring, $52^{\circ}$ North, Münster, Germany

Software required: Java runtime environment

Program language: Java

Availability and cost: Users can access binaries and source code freely

Documentation and code available at:

http://52North.org/sid

Name of software: SID Creator

Developers: $52^{\circ}$ North $\mathrm{GmbH}$

Contact information: Arne Bröring, $52^{\circ}$ North, Münster, Germany

Software required: Java runtime environment

Program language: Java

Availability and cost: Users can access binaries and source code freely

Documentation and code available at:

http://52North.org/sid 


\section{Introduction}

The availability of spatio-temporal data and reliable environmental models is necessary to effectively monitor natural resources and to foster sustainable development. For instance, within the forestry domain, early warning systems based on spatio-temporal data and modelling tools are required for the successful monitoring of forestry resources and to assess their vulnerability towards natural hazards. In this context, the availability of interoperable data and software, based on common standards, is important for their reusability and efficiency.

In operational forest fire monitoring, models are used to calculate fire danger indices based on a great diversity of spatio-temporal data (Camia et al. 2006). The availability of high spatio-temporal resolution data improves the accuracy of the results of such models, significantly. The European Forest Fire Information System (EFFIS) uses the fire danger model to produce the Fire Weather Index (Camia and Amatulli, 2010) and to build forecast maps at a pan-European scale. To calculate such fire weather indices for a higher spatiotemporal resolution and to produce maps at local scale, the model needs to be run, among others, with meteorological data provided by local in-situ sensors. Thus, the availability of this data through interoperable components is crucial for the model to be reused across different geographic areas.

Nowadays, operating these environmental models has shifted from centralized applications towards distributed infrastructures (Mineter et al., 2003). Geospatial Web services, deployed in Geospatial Information Infrastructures (GII), are combined to feed and calculate environmental models with up-to-date data (Nebert, 2004). In recent years, this approach has been demonstrated to be effective in various domains such as urban planning (Bishop et al., 2000), forestry (Davis et al., 2009), risk management (FriisChristensen et al., 2009) and hydrology (Granell et al., 2010).

Despite the known benefits of GIIs based on standardized services for geodata provisioning (Lee \& Percivall, 2008), the variety and complexity of data encodings, as well as Web service interfaces, requires considerable efforts to publish new content (Díaz et al, 2011). In consequence, data often remains only locally available, in proprietary formats, without being accessible in an interoperable way. Overall, there is a scarcity of data in GIIs today (Díaz et al, 2011) and in particular of sensor data. In the case of sensor data, the various native protocols of sensors are defined by their manufacturers, since standard encodings for sensor protocols are not widely accepted. To integrate such sensor data with a GII, the raw sensor data needs to be translated to the standardized protocols of a GII. So far, this is done by manually implementing adapters for each sensor type, resulting in extensive efforts when developing large-scale systems (Aberer 2006). This problem is similar to the issue one faces when a data file, e.g. comma separated values (CSV), needs to be interpreted, without the inherent description of data columns.

This paper describes an approach for interoperable data publication that closes the gap between GII standards and the need for sharing sensor data. We present a GII approach which advances the state of the art by adding the capacity of publishing raw in-situ sensor data into GII services to make them available as higher-level observations. To demonstrate our work we run an experiment of such an enhanced GII in fire danger modelling. We discuss how, we increase the availability of these high spatio-temporal resolution observations, to improve the accuracy of environmental models with the final goal of making use of these observations in a forest fire danger scenario to improve the monitoring of forestry resources. In this work, a publication service called the GEOSS Service Factory (GSF) (Díaz and Schade 2011) is used as a middleware component, which in combination with the Sensor Interface Descriptor (SID) (Bröring et al. 2010a), provides a single and standards-based entry point to facilitate the publication of sensor data into 
GIIs. The approach that is presented within this paper is applied to integrate real-time meteorological data coming from in-situ weather sensors for a fire danger model scenario from EFFIS.

Section 2 provides an overview on the related work and we introduce relevant concepts such as GIIs, the Sensor Web, as well as fire information models. Section 3 presents the foundations to publish sensor observations in a GII to increase the availability of this data as interoperable services in order to improve the accuracy of fire danger models. Section 4 describes our approach, while Section 5 applies the approach in a use case on fire danger assessment. Finally, Section 6 summarizes the presented findings and outlines future work.

\section{Background and Related Work}

We aim at increasing the availability of high spatio-temporal resolution sensor observations for running models and improving their accuracy for better environmental monitoring. Therefore, our approach extends the architecture of a GII with two components that allow users to publish these sensor observations. In this section, we outline relevant concepts of GIIs and their applicability for environmental monitoring. Furthermore, we describe standards and related work on data sharing, with a special focus on sensor data. Finally, we describe related research on fire information models since it is used as our case study.

\subsection{Geospatial Information Infrastructures and Web Services for Environmental Monitoring}

GIIs are based on specific technologies, policies and institutional arrangements for sharing geospatial data and computing models between different stakeholders (Masser, 2005). Government mandates, such as the INSPIRE European Directive (INSPIRE, 2007) recommend standards and policies for improving the sharing of resources and environmental (and related) decision-making. GIIs have been identified as required tools to realize more dynamic systems for integrating new sources of information and improving decision making (Craglia et al., 2008). In case of environmental monitoring, such as forest fire monitoring, it is essential to have modelling systems in place at different spatial resolution (de Groot et al., 2006) to assess and limit, for instance, the fire impact. In recent years, different initiatives have emerged at different administrative levels (regional, national, international) to organise the geospatial information in general and environmental information in particular in the realm of GIIs. These include: the INSPIRE, the Global Monitoring for Environment and Security (GMES) initiative and Shared Environmental Information System (SEIS) on European level, and Global Earth Observation System of Systems (GEOSS) at global level. The purpose of the GEOSS initiative is to achieve comprehensive and coordinated observations of the Earth to improve monitoring and enhance prediction of the behaviour of the Earth (Cristian, 2005; Lautenbacher, 2006). INSPIRE, GMES and SEIS can be seen as part of the European contribution to GEOSS.

Those initiatives propose the deployment of information infrastructures, which rely on distributed Web services that provide the required functionality. For instance, INSPIRE specifies, at conceptual level, services to support discovery, view, download, transformation and invocation capabilities. The technical level provides a range of interoperability standards for the integration of the information infrastructures and components (Mykkänen, and Tuomainen, 2008).

In GIIs, interoperability is ensured to a large extent through standards governed by the Open Geospatial Consortium (OGC). OGC has published several standards, which promote 
syntactic interoperability through the use of Web services (Percival, 2008). The existing specifications provide a baseline for GII such as for INSPIRE; these include for instance the OGC Catalogue Service (CS-W) (Nebert and Whiteside, 2004) and the more recent interfaces of the Sensor Web Enablement initiative (SWE1). Other specifications such as the OGC Web Processing Service (WPS) (Schut, 2007) provide an interface for offering processing functionality as distributed Web services (Foerster et al, 2011). The publication service, described in this work, utilizes those standards to publish data in standards-based services, to increase data interoperability. Moreover the developed publication service itself is implemented with the WPS interface to offer the publication capability of diverse data (here in particular sensor data) into GII.

\subsection{From Sensors to the Sensor Web}

Today, there is a great variety of environmental sensors used out in the field. Thereby, such sensors observe all aspects of our environment: air, water, soil, but also human activity (e.g. traffic). To integrate sensors and their measured data with information infrastructures, there are typically multiple intermediary stages, rather than a direct link. After connecting a sensor (or sensor network) with a data acquisition system, several integration steps customized for a specific information infrastructure are implemented (Figure 1). Those integration steps are often specifically designed for one organization. Then, the data acquisition system either polls data from the sensors or receives data which are autonomously sent by the sensors. Sensors transmit the data over a particular transmission protocol. This is often a wired serial connection, such as RS-232, but can also be Ethernet, or even a wireless connection (e.g. Bluetooth).

To overcome the need for customized, and cumbersome, sensor integration, this work develops a standards-based approach usable by sensor network providers. While most environmental sensors today are still connected to the data acquisition system via a serial connection, the here developed approach requires an (at least indirect) Ethernet connection and an IP address for each sensor. To equip serial connected sensors with an Ethernet connection and an IP address, the sensor provider can establish a serial-toEthernet bridge which can be done with existing software ${ }^{2}$. We take this as a valid assumption, since also current research goes into this direction under the umbrella of the Internet of Things research field (Gershenfeld et al., 2004) and by means of emerging technologies such as IPv6 (Hui and Culler, 2008).

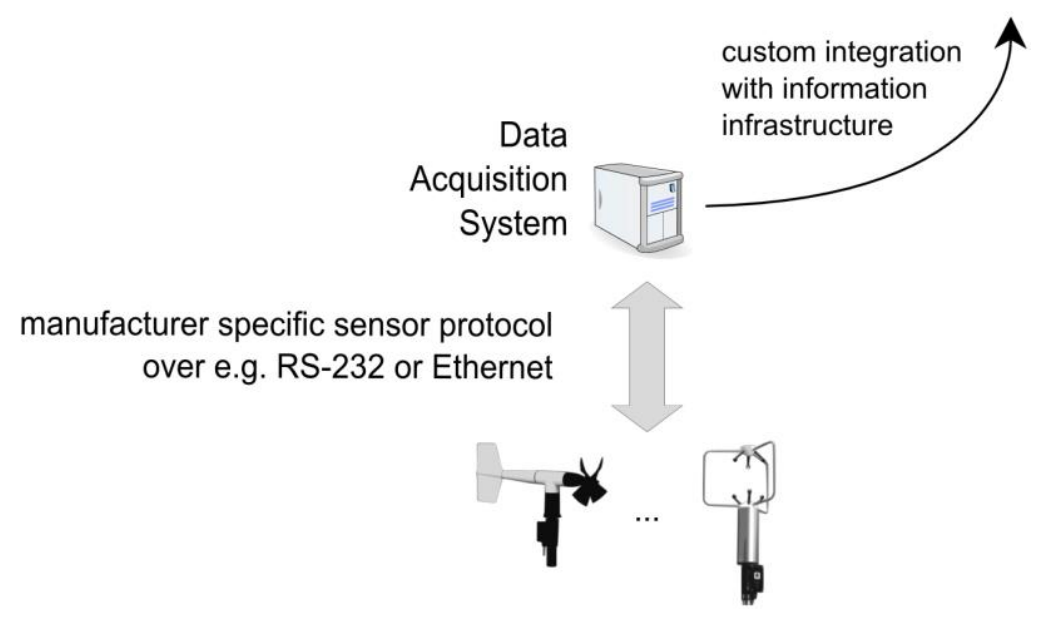

Figure 1. Custom integration of sensors with information infrastructures.

\footnotetext{
${ }^{1}$ http://www.opengeospatial.org/ogc/markets-technologies/swe

${ }^{2}$ For example, Digi PortServer TS (http://www.digi.com/products/serialservers/portserverts) can be used.
} 
To achieve our goal of a standards-based approach for integrating sensors, well-defined Web interfaces are needed for enabling interoperable access and hiding the heterogeneous protocols of environmental sensors. For this purpose, the so-called Sensor Web has been developed. It enables Web-based discovery, exchange and processing of sensor observations, as well as task planning of sensor systems (Nittel 2008). The SWE initiative of the OGC defines standards, which can be utilized to build such a Sensor Web (Botts 2008). SWE standards make sensors available over the Web through standardized formats and Web service interfaces by hiding the sensor communication details and the heterogeneous sensor protocols from the application layer (Bröring 2011a). The SWE framework defines multiple Web service interfaces to provide the functionality of sensors on the Web. Of core interest for this work is the Sensor Observation Service (SOS) (Na and Priest, 2007, Bröring et al, 2012). The SOS provides interoperable access to real time sensor data as well as sensor metadata. Therefore, it defines operations to register new sensors, insert their observations, and retrieve those observations and associated information resources by supporting flexible spatio-temporal query filters. Thereby, the SOS encodes observed sensor data compliant to the Observations \& Measurements (O\&M) standard (Cox 2007). The metadata descriptions of sensors are encoded conform to the Sensor Model Language (SensorML) specification (Botts 2007).

SensorML defines a schema for sensor related processes such as measuring or post processing procedures. Physical as well as logical sensors are modelled as processes. The functional model of a process can be described in detail, including its identification, classification, inputs, outputs, parameters, and characteristics such as a spatial or temporal description.

O\&M defines a schema for observations. An observation has a result (e.g. $35^{\circ} \mathrm{C}$ ) which is an estimated value of an observed property (e.g. temperature). This observed property is a characteristic of a feature of interest (e.g. the Black Forest, Germany). The result value is generated by a procedure, e.g., a sensor such as a thermometer described in SensorML. These four central components are linked within SWE.

\subsection{Publishing Sensor Observations in GII Services}

Bridging the vast heterogeneity of mostly vendor-specific sensor protocols and the coherent information infrastructures, such as GIIs, can be addressed from two directions. On the one hand, different standardization initiatives try to establish interoperable interfaces on the sensor level. Here, the IEEE 1451 family of standards (http://ieee1451.nist.gov) seems most promising. IEEE 1451 is a universal approach to connect sensors to diverse networks and systems. However, in today's real world applications not only IEEE 1451 but in fact a huge variety of sensor interfaces (standardized or proprietary) are utilized. Hence, several projects tackle the issue from by abstracting from the variety of sensor protocols. An example is the AnySen project (Klopfer 2009) that is capable of reading and interpreting data from sensor nodes by abstracting the sensor protocols and reading the sensor description from an external file. Walter \& Nash (Walter 2009) analyze system models, which lower the implementation barrier for coupling sensor systems and SWE services. The authors suggest lightweight SWE connectors, which can be adapted to different raw sensor formats to convert them to SWE-based data models. The Sensor Abstraction Layer (Gigan 2007) makes use of SensorML to describe sensor interfaces. As a library, it offers high-level functions to access sensors by hiding their specific technological details. Missing are mechanisms for the final connection to SWE services.

Similar to the Sensor Abstraction Layer approach is the Sensor Interface Descriptor (SID) concept (Bröring et al. 2010a, Bröring et al. 2011d) which enables the declarative 
description of sensor interfaces, including the definition of the communication protocol, sensor commands, processing steps and metadata association. The model is designed as a profile and extension of the SensorML standard. Based on this model, SID interpreters can be built in order to be able to translate between sensor protocol and application protocols (e.g. SWE). Such interpreters for SID instances can be created independently of particular sensor technology. They establish the connection to a sensor and are able to communicate with it by using the sensor protocol definition of the SID. Instances of the SID model designed for a particular sensor type can be reused in different scenarios and can be shared among user communities.

In addition to the heterogeneity of sensor data encodings, the second issue in publishing sensor data into GIIs is to deal with GII publishing mechanisms. GIIs are traditionally topdown infrastructures that do not consider active participation and, the complex publication mechanism assumes the underlying understanding of the standard service specifications and their implementations (Díaz et al, 2011). ICT experts became the only mediator between the environmental experts and data providers, who create and understand the content, and the infrastructure. To improve the given situation, (Díaz et al, 2011) presented a distributed architecture based on INSPIRE principles and extended it with a service framework component. This component improved ad hoc integration and publication of geospatial data resources within GIIs focusing on user-generated content. The service framework addresses the need to improve the availability of geospatial data resources by providing user-driven mechanisms to assist users in wrapping resources to generate INSPIRE-based services increasing the capacity building in GIIs. Díaz and Schade (2011) outlined the extension of this approach to migrate the publication functionality to a Web service implemented with the WPS interface. This publication service, the GSF, aimed to assist in the publication of content within existing GII.

In the scope of this work, we continue with this hybrid GII building methodologies, where according to existing standards and protocols we assist users in participating and publishing content in GII. We extend the GSF approach to combine the assisted publication in existing GII nodes with the semi-automatic interpretation of sensor data streams. In this sense, the GSF is combined with the SID interpreter to support the publication of sensor observations in existing GIIs. Combined, the GSF and SID concepts facilitate the provision of near-real time sensor data and will eventually increase the availability of data via standardized GII services.

\subsection{Forest Fire Information Systems and Models}

Forest fire Information Systems (FIS) provide information on the vulnerability and impact of fire events in forest areas. They specifically provide access to real-time, spatially referenced information on climatic conditions and the assessment of fire danger within a geographic area. Such systems also provide a means of systematically evaluating and combining parameters that are used to determine the ease of a fire starting and/or spreading within a landscape (Lee et al. 2002). At a more general level, they can be considered as spatial decision support systems, that expert users rely on for the management of natural resources in areas susceptible to wild land fires, such as in Mediterranean Europe, parts of the United States of America and Australia. Forest fire information systems commonly provide weekly forecasts and fire predictions that are updated on a daily basis.

FIS are underpinned by sophisticated forest fire modelling capabilities that rely on a range of meteorological, topographic and spatially referenced forest inventory information. There exist two main types of forest fire models, fire danger forecast models and forest fire behaviour models Arroyo et al. (2008). The former relate to the production of fire danger forecasts using meteorological and topographic variables and provide information 
on the potential forest fire danger. Some examples of these models include the Australian McArthur Forest Fire Danger Rating System (McArthur, 1966), the Portuguese Index, the Spanish ICONA method (Camia and Bovio, 2000) and the Canadian Fire Weather Index (FWI) (van Wagner, 1987). The latter of which has been adapted to European conditions and is used operationally in EFFIS (Camia et al. 2006).

Forest fire behaviour models are used to simulate the growth and geographic spread of forest fires (Vasconcelos and Geurtin, 1992). They typically combine fuels maps (e.g. timber or vegetation structure and quantity) with topographic and meteorological variables in a spatial modelling framework to estimate parameters such as the direction of fire spread, heat per unit area, fire line intensity and flame length. In this regard, Arroyo et al. (2008) noted that a plethora of fire models have been developed by Forest Authorities around the world, which have been adapted to national and/or local conditions. Nonethelss, the model developed by Rothermel (1972) to simulate fire spread in surface fuels remains one of the most widely used models.

It is evident that the core input into these fire models is meteorological data and clearly, the ability to harness such information at higher frequencies and spatial resolutions could significantly benefit the calibration and resolution of the predictions. One of the few examples, is the Croatian iForestFire system, which harnesses meteorological data from a sensor network to provide a more accurate forest fire warning system in Croatia (Stipaničev, 2007; Šerić et al. 2011). In this work, Section 5.2 deals with the description of a Fire Danger model and how the accuracy of its results can be improved by our approach, since we address the availability and interoperable access of high spatio-temporal resolution sensor data, in particular meteorological data.

\subsection{Fire Danger Model}

For this paper, we demonstrate our approach based on the use case of EFFIS and its fire danger model that produces the Fire Weather Index forecast maps at a pan-European scale. These maps are computed on a daily basis providing a harmonised forecast of the fire danger based on five classes. The core inputs to the model are pan-European climate data from Meteo-France and the German Meteorological Service (DWD) (Camia et al. 2006). The forecast data are used to generate maps with a spatial resolution of $45 \mathrm{~km}$ for Meteo-France and $36 \mathrm{~km}$ based on the data from DWD. In short, it represents the intensity of the propagating flame front depending on the quantity of energy released from a linear unit of the front itself. Currently, the Fire Danger model computes the FWI on a daily basis using the following meteorological variables:

1. Temperature at noon $\left({ }^{\circ} \mathrm{C}\right)$;

2. Relative Humidity at noon (\%);

3. Wind speed at noon $(\mathrm{km} / \mathrm{h})$;

4. Daily rain $(\mathrm{mm})$.

The resulting FWI is a composite index of five sub-indices that refer directly to the daily variations of water content for fuels, the propagation speed and the expected intensity of the flame front. The following list summarises the sub-indices and more detailed information regarding their calculation can be found in Van Wagner and Pickett (1987); Van Wagner (1987), while Figure 11 presents the schematic structure of the FWI and its components: 
a. Fine Fuel Moisture Code (FFMC) which is the expression of the water content of litter and fine dead fuels. It provides a direct indication of the ignition and flammability of fine dead fuels;

b. Duff Moisture Code (DMC) represents the water content of a moderately thick organic layer.

c. Drought Code (DC) represents a rating of the water content of a deep, compact organic layer in the soil and provides a good indicator of seasonal drought;

d. Initial Spread Index (ISI) provides an estimate of the expected propagation of the flame front;

e. Build Up Index (BUI) represents a rating of the total fuel available for burning.

Section 5 will illustrate how this model is used to validate the approach described in this paper. By improving the availability of standardized sensor observations at different scales, this model will be run with different datasets to produce more localised fire danger forecasts.

\section{Publishing Sensor Observations into GII}

GIIs stimulate the use of standard formats and exchange protocols, to permit the distribution of geospatial content and functionalities to interested users (Granell et al, 2010 ) by means of Web services. In this section, we describe an approach, which, extending a standard-based service architecture aligned with INSPIRE principles, goes beyond the traditional functionality and enables to publish and integrate geospatial content such as sensor observations. Our approach applies the combination of two components deployed in the extended architecture. We describe how the GSF and the SID concepts are combined for a flexible translation of sensor data to publish them as sensor observations in a GII. We outline the anatomy of GSF and SID to describe their linkage that lowers the barrier of integrating heterogeneous sensor data encodings.

\subsection{Service Architecture Overview}

The INSPIRE technical architecture describes aspects such as service types and their interfaces. This three-layered architecture differentiates the 'Application' layer, the 'Geospatial Networking Service' layer, and the 'Geospatial Content' layer (Figure 2). It provides the required functionality through a set of services deployed in the services layer. Users access this functionality through the 'Application' layer that includes end user applications. The services implement standard-based interfaces to provide an interoperable channel to the content deployed in the 'Geospatial Content' layer. In Figure 2 , we can see the services functionality considered by the INSPIRE directive: Discovery, View, Download and Invocation. In addition, Figure 2 shows an augmented INSPIRE architecture, which provides a new service type: the Publication Service. With this new service, we aim at assisting users in publishing content to maintain GIIs and increase the availability of updated content (Díaz and Schade, 2011). In this work, our approach combines this new service with the SID concept, to publish high spatio-temporal resolution sensor observations to Sensor Observation Services as part of INSPIRE-based GIIs, so they are available to be integrated in environmental models. 


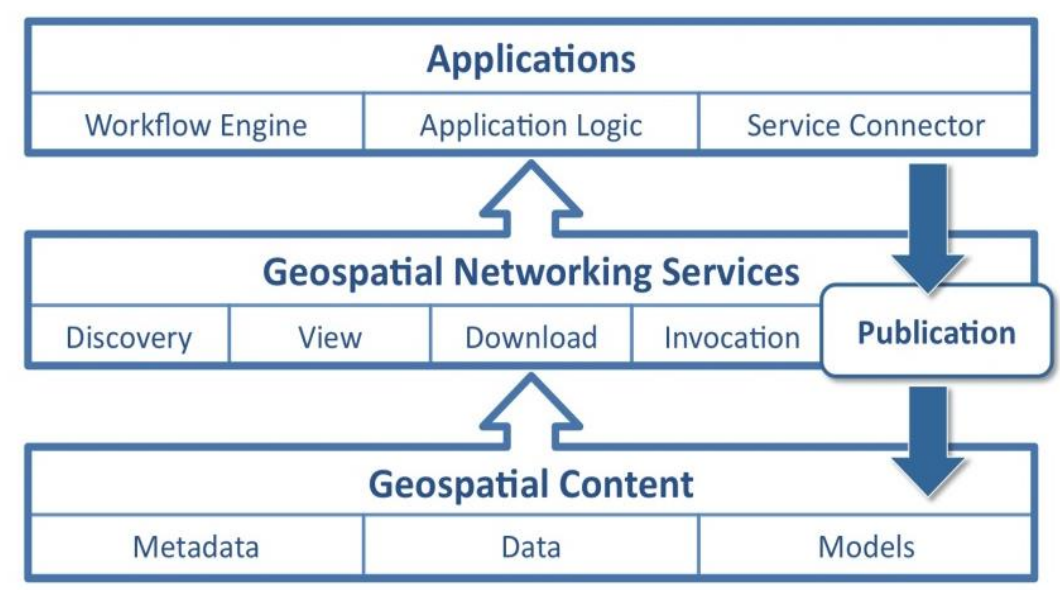

Figure 2. Conceptual architecture of an INSPIRE-based Geospatial Information Infrastructure extended with our approach for content publication.

\subsection{GEOSS Service Factory (GSF)}

We have introduced a new service type that provides publication capacities; the implementation of this work is the GEOSS Service Factory (GSF). As part of a GII the GSF assists in the publication of different types of content in existing standards-based service instances. The GSF prepares a channel that hides complexity from the user who aims at publishing content, and thus, it facilitates the sharing of content, while remaining loyal to the standards of geospatial initiatives to reach the required level of interoperability.

The features of the GSF are: (i) its behaviour is modelled with the Abstract Factory software design pattern (Gamma et al, 1995) to make it more scalable. This pattern is a creational pattern used to instantiate new entities. It encapsulates a group of individual factories that have a common topic. In our case, the GSF holds a group of factories providing operations to publish new content entries in Geospatial Services and (ii) the GSF is a Web service that implements the OGC WPS specification, thus offers the publication capacity as a standard-interfaced process. Díaz and Schade (2011) provide a deep discussion on the conceptual architecture and implementation details of the GSF.

Figure 3 shows the architecture of the GSF and its main components. The basic idea is that the GSF publishes content by creating new data sources in existing services. It is composed by different factories each of them dealing with the publication of content in a different service type. Besides those factories the GSF contains two other modules: The Metadata Generator (MDGenerator in the figure) is able to generate a small discovery-purpose metadata of the published content. The Transformation Service deals with data format transformation. This module prepares data format and encoding to generate appropriated formats, which can be handled by the existing services as data storages.

As shown in Figure 3, when the GSF is invoked (step 1), the content to be published is sent as an input parameter. The GSF settings are stored in the PublicationProfile, which reflects the publication policy of the GII (step 2). This XML-based document can be set as a default configuration file or sent as an input parameter. It states to which type of service the content is going to be published and where the service instance is running. Then the GSF delegates the content to the corresponding factory or factories that are configured to deal with this content type (step 3). Next, the factories communicate and publish content to the existing services (step 4 and 5) via the service's application programming interface (API), where in our context, most of them implement OGC interfaces, but other service specifications can be added. 


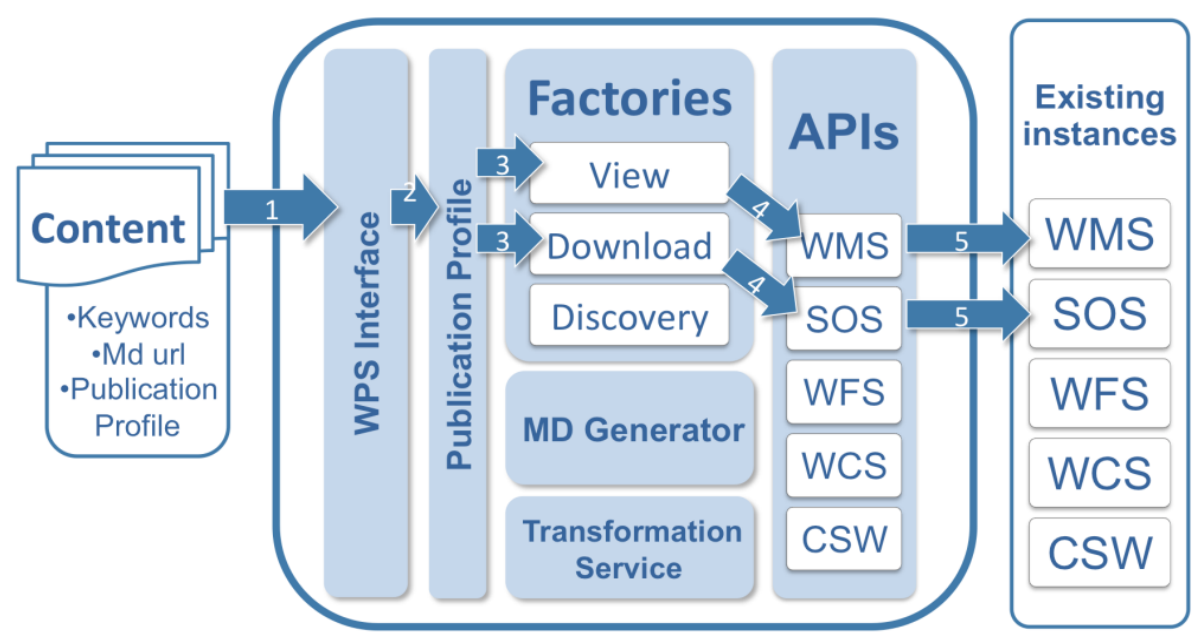

Figure 3. GSF components overview.

After the content is published, the GSF triggers the Discovery Factory to register the content in an open Catalogue. In this case the Discovery Factory, through the MDGenerator, queries the updated services to extract discovery-related metadata. The MDGenerator extracts the metadata provided by the services which contains among other things, information about contact info, data format, scale, title, boundary box, and the link to retrieve the data through this service. This metadata are transformed to metadata according to ISO format (ISO19115, 2003). Afterwards, the metadata is published in a catalogue service for further discovery. The output of the publish process is a link to the metadata entry containing the information of the content and its accessibility.

To enable the GSF for publishing sensor data, we introduce in the next section the Sensor Interface Descriptor (SID) concept. Finally, SID and GSF are connected through the Transformation Service module to collaborate and publish sensor observations to existing GII nodes, as described in Section 3.4

\subsection{Sensor Interface Descriptors (SID)}

To retrieve and process data from an arbitrary set of sensors, users need to understand a wide variety of sensor protocols. Such protocols define the commands for retrieving data from an instrument as well as the format in which the data are transmitted. The principal problem is that a wide variety of protocol designs exist with varying levels of complexity tailored for the specific instrument, however, no established protocol standards are used by the various vendors. Data can be transmitted in ASCII format, where data tokens are separated by comma or tab characters. Instead of such delimiter signs, a binary encoding may use fixed positions for certain fields within a data block, as it is the case for example for the protocol of the HOBI Labs HydroScat oceanographic fluorometer (HOBILabs 2008). Increasing the complexity, an instrument may even use multiple formats for different data blocks within the same stream.

Similar to the variety of data formats, the definitions of sensor commands vary among instruments. For example, some instruments start sending data right after being switched on without external triggering. Others start a continuous sending process after receiving an according command (e.g., the marine conductivity, temperature and pressure sensor Seabird SBE-37SM (Sea-Bird-Electronics 2010), and others need such a command for every data transfer request (e.g., the Vaisala weather station considered in Section 5.1). 
Interpreting and processing the protocol after connecting to a sensor usually requires the implementation of an according driver that conforms to the sensor protocol specification and a customized integration with the information infrastructure (see Section 2.2). Taking this burden from users of sensor devices, Sensor Interface Descriptors (SID) provide a way of describing sensor protocols in a standardized way. Although not every possible variation is supported, the SID model can be applied to a wide variety of protocols.

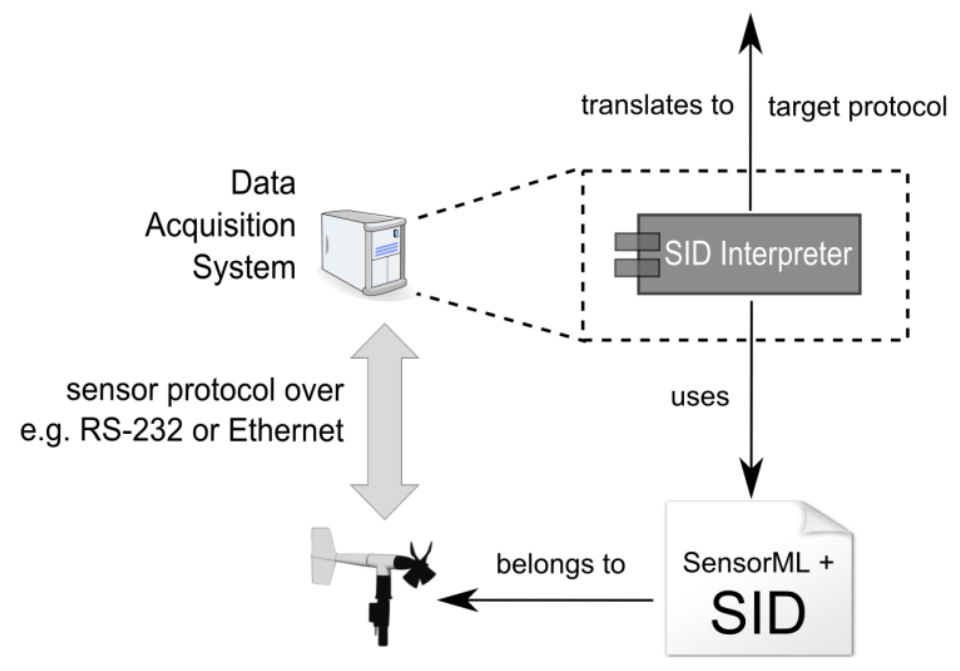

Figure 4. Translation of sensor protocol by an SID interpreter.

The SID concept and model are described in detail by Bröring et al. (2010a). The architectural principle of a system infrastructure incorporating SID is illustrated in Figure 4 , an extension of Figure 1. A sensor communicates with a data acquisition system in its native sensor protocol over a transmission technology such as RS232 or Ethernet. This sensor can also act as a sensor gateway (network sink) so that other nodes of a (possibly mobile) sensor network communicate with it. The SID interpreter runs on the data acquisition system and uses SID instances for the different sensors to translate between the native sensor and the target protocols and data models, e.g., the O\&M data model (Section 2.2). An example of an SID instance describing the data model of a weather station is given in Section 5.2

\section{Approach for an Assisted Publication of Sensor Observations into GII}

The GSF as a publication service in combination with the SID to interpret proprietary data offer together a powerful tool to facilitate the whole workflow of integrating sensor data into GII. This way, sensor data becomes available in an interoperable manner and can be integrated with other geospatial information for further analysis, e.g., fire danger assessment.

Figure 5 shows the components of the Geospatial Networking Services layer (Figure 2). Deployed in this layer, different service types implement OGC service specifications to provide required functionality. We extend the functionality by adding the GSF as a Publication service. The GSF implements the WPS interface (see top of the Figure 2). The GSF can be invoked by a sensor provider via the Execute operation of the WPS to initiate the publication of the sensor and its data. When calling the Execute operation, a sensor 
provider transmits (1) the SID file of the sensor and (2) the sensor's IP address ${ }^{3}$ as input parameters as shown in Figure 6. The GSF, by means of the Transformation Service, hands this information over to the SID Interpreter which parses the SID file (using the internal SID Parser component) and sends commands to the sensor to deliver its captured data. To physically connect to the sensor, the internal Data Source Connector component is utilized. Once the sensor data stream is opened, the SID interpreter transforms the incoming data from the native sensor protocol to $0 \& \mathrm{M}$ based on the interface description contained in the SID file and using its Protocol Transformer component. In our use case, these sensor observations are published by means of the Download Factory to a standard Sensor Observation Service (SOS) (Section 2.3), since its interface is most suitable for providing sensor observations to a fire danger model.

The GSF is able to publish geospatial content to different service types, to do so its factories are able to deal with these service types and the interfaces that they implement such as the SOS or WFS. In order to publish O\&M observations into a SOS service, the Download Factory is in invoked by the GSF (Figure 5). When it is specified that these observations are published for visualization, the View Factory publishes them in a WMS and metadata (automatically generated by the MD Generator) are published in a Catalogue service (CSW) by the Discovery Factory.

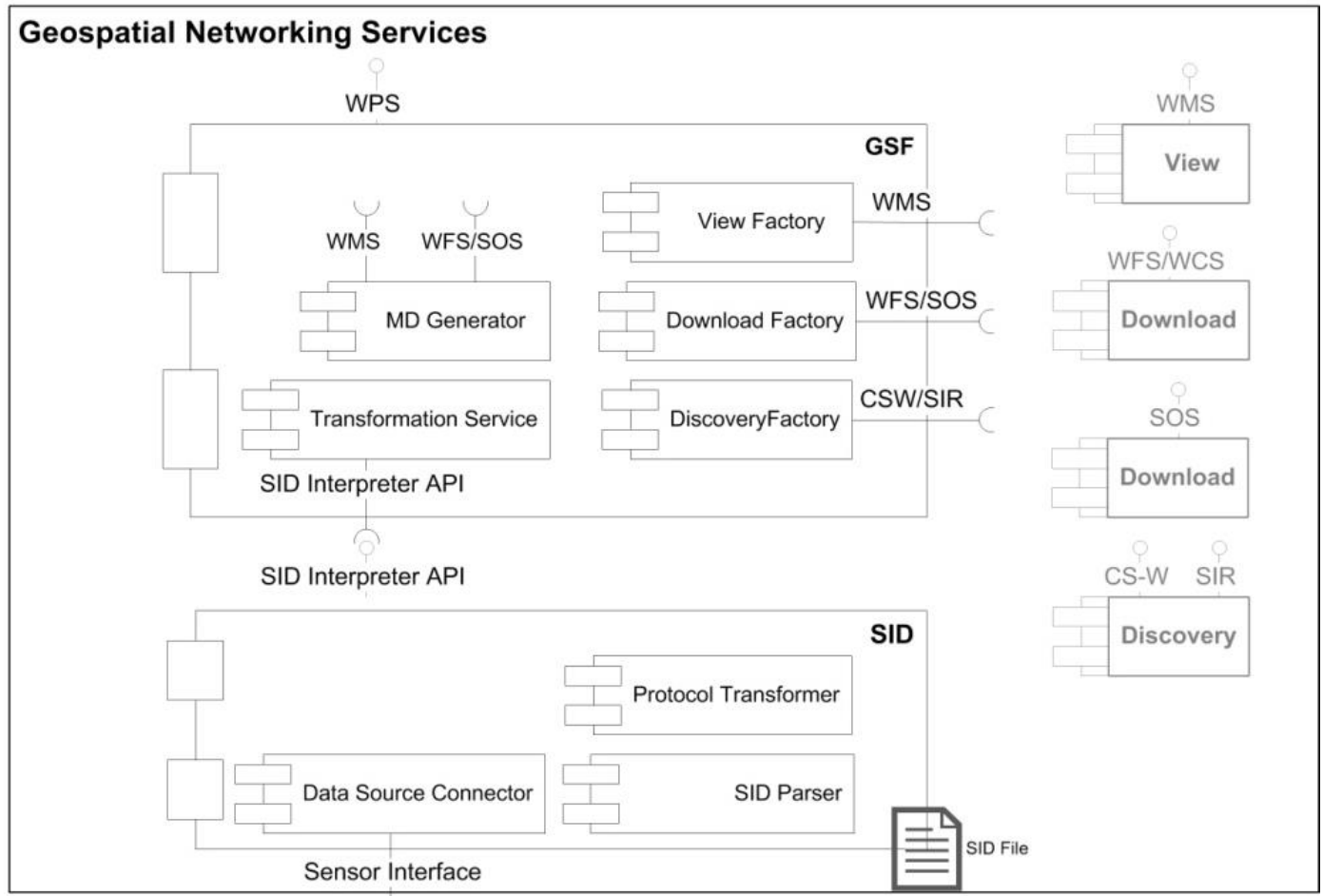

Figure 5. Detail of the Service Model for sensor data interpretation and publication in GII (in UML notation)

To illustrate the developed approach, Figure 6 shows a UML sequence diagram with all participants involved in the publication of sensor data into GII services. Firstly, the user (usually the sensor provider or administrator) invokes the GSF via its WPS interface. Listing 4 shows an example of a WPS Execute request to be sent to the GSF for triggering

$3 \quad$ As already stated in Section 2.2, in this work, we require that the sensor has an (at least indirect) Ethernet connection and an IP address so that the GSF service (and the associated SID interpreter) can access the sensor remotely. 
the publish process. The Execute request contains the SID file as well as the IP address of the sensor.

Secondly, the Transformation Service triggers the SID Interpreter which then sends a "get data command" to the sensor so that the sensor data stream is opened up, the SID interprets the stream of incoming sensor data and transforms it to the corresponding O\&M encoded data that will be retrieved by the Transformation Service as part of the GSF.

Thirdly, the streamed sensor data are in a known format, namely encoded as 0\&M observations, and ready to be published to an SOS. The GSF sends those observations to the Download Factory. This factory internally uses the SOS API to connect to a concrete SOS instance and publishes the sensor observations there. In this case the publication is performed by sending an InsertObservation request according to the SOS specification. That way, the incoming sensor data stream is successively uploaded to an SOS, where it is stored and henceforth available in an interoperable way.

Fourthly, to increase the visibility of the published data within the GII, metadata needs to be published in a Catalogue Service, which is used for discovery purposes. To perform this step the GSF utilises its internal MD Generator which requests the updated services (here, the SOS instance) to retrieve service and data metadata, this metadata information is be transformed to a standard metadata format (IS019115, 2003) that contains the information about where and how the sensor data are available. Finally, the Discovery Factory publishes the metadata in a Catalogue Service according to the CSW specification. The link to this metadata available in the Catalogue Service is returned to the user. To be noted is the fact that no secure connections are provided to these components, we are aware of the limitation of these components in order to avoid undesired connections. The secure access is an issue that we maintain out of the scope of this paper but it will be considered in the near future as one of our research lines.

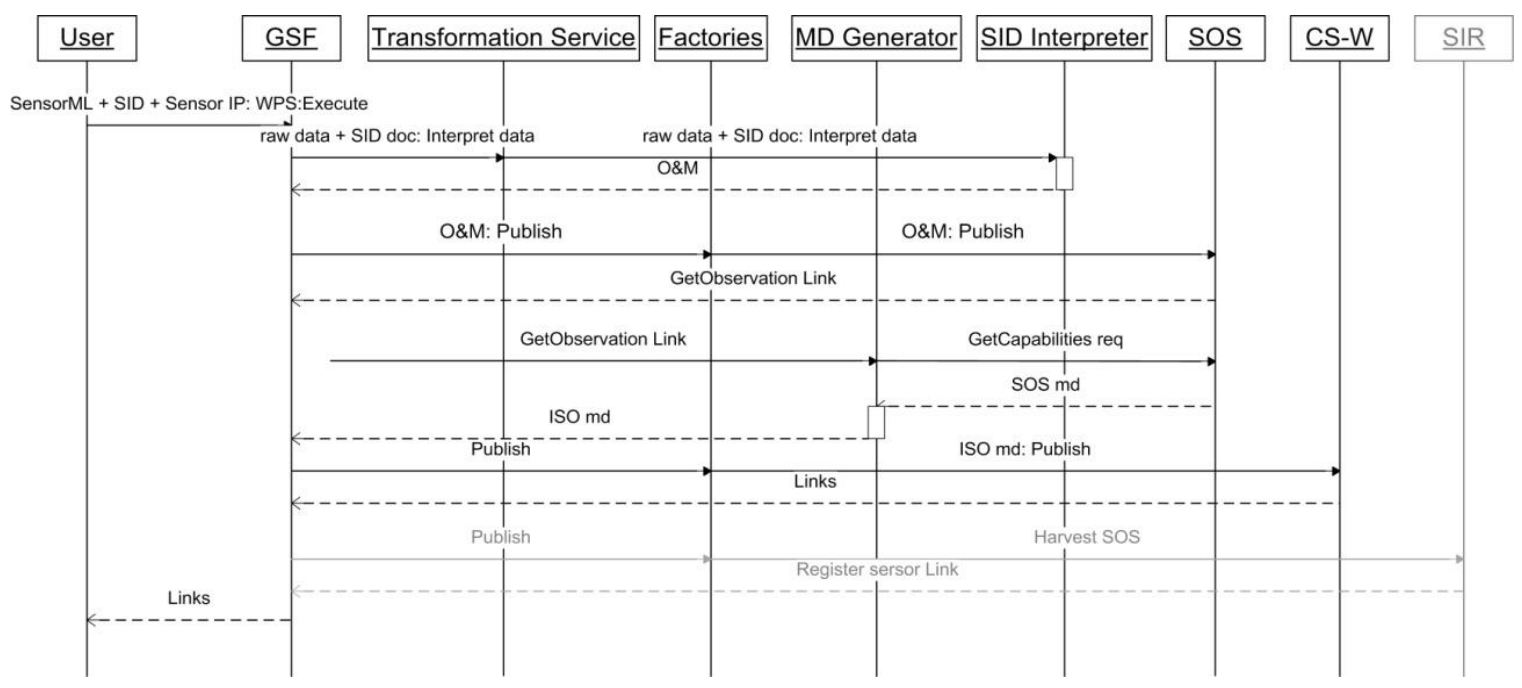

Figure 6. Sequence diagram for sensor data publication and registration into GIIs.

In order to perform a qualitative assessment, Figure 7 compares the proprietary way of integrating sensor data with the here presented standards-based approach. The proprietary way requires the manual implementation and configuration of sensor driver software to integrate a sensor with the existing infrastructure, which, in turn, has to be manually adapted to the Sensor Web services, such as the SOS. Both kinds of adaptation efforts are specific to the internal infrastructure of the organization and are hence not shareable with others. The standards-based approach is shown on the right side of the 
figure. The adaptation to the Sensor Web services is automated through GSF and SID Interpreter. To integrate a sensor, SIDs can be reused, since an SID is defined for a sensor type. In future, SIDs can for example be provided by a sensor manufacturer or third party projects. If none can be found, an SID can be semi-automatically created using the SID Creator (Bröring et al. 2011c). This shows that the key advantage of the developed approach is its grounding in standards and the openly defined specifications which makes compliant components reusable and shareable. In the future, the development of supporting tools which rely on the developed approach is a key to further automate the sensor integration process.

\section{Proprietary Approach Standards-based Approach}

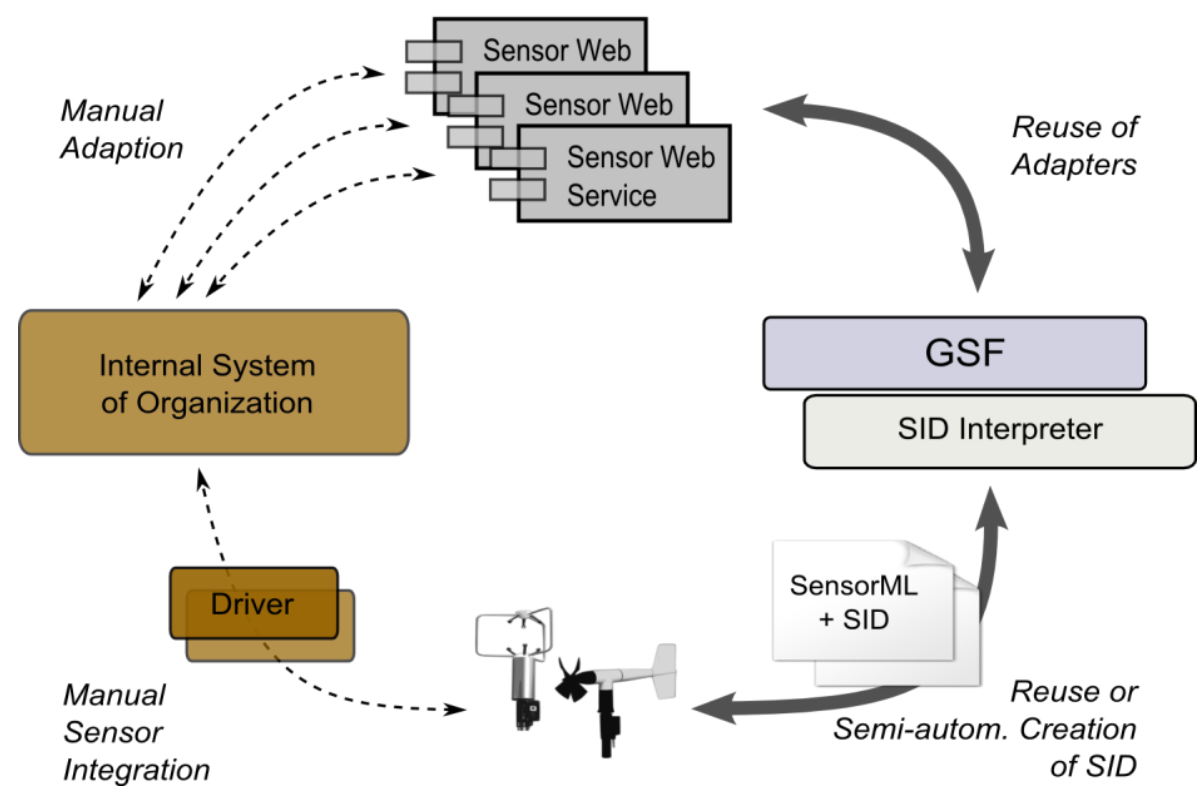

Figure 7. Comparison between proprietary and standard-base publication methods (based on Bröring, 2012a).

\section{Use case: Fire Danger Assessment}

Fire danger assessments are crucial parts of an early warning fire system and are needed to reduce the potential deleterious impacts of forest fires. Fire danger models produce results whose quality depends not only on the underlying algorithm but also on the input data used (Heuvelink, 1998). The accuracy of the results is tightly coupled with the accuracy and the scale of the input datasets. This work aims at increasing the availability of high spatial scale sensor data as standards-based services to feed scientific models and produce more accurate results. The bottom part of Figure 8 shows the current status of the Fire Danger Model, where European scale data, among others, meteorological data, is used as input parameters to produce European scale FWI. The top part of the figure, illustrates how our approach, assisting users in geospatial content publication, and more specifically in the publication of (meteorological) sensor data, can improve the availability of these data as standard services, so it can be reuse as inputs of the model to produce more accurate results. Therefore, sensor data, available at national or local scale through SOS-implemented services, can be reused as input data into forest fire danger models. These model results can be used to produce national or local fire danger maps with a higher resolution. 


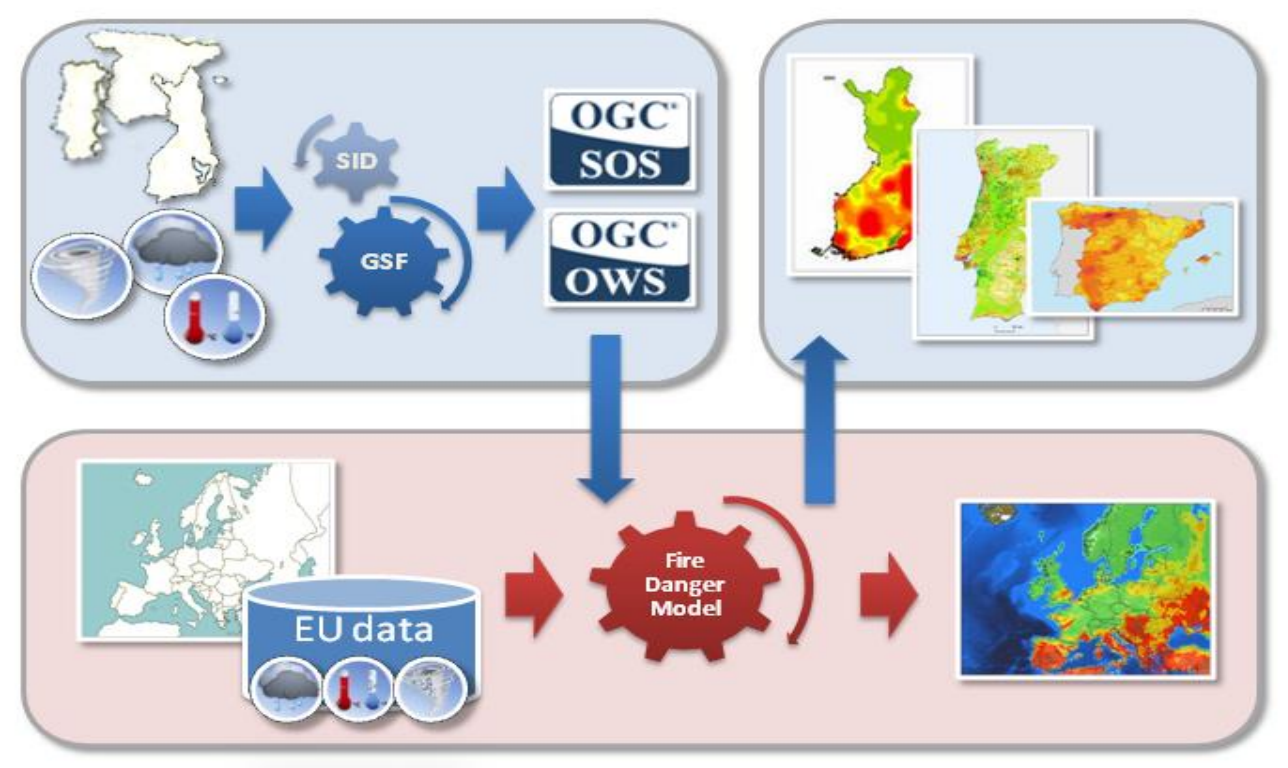

Figure 8. Applicability of GSF_SID into Fire Danger Model execution.

\subsection{Publication of Meteorological Sensor Data}

By means of the above described architecture (Section 3), we integrate a Vaisala WXT520 sensor system (Figure 9) as an example for a common and widely used weather station and present how its data can be published in GII services. This sensor system is capable of measuring the parameters (e.g., air temperature and accumulated rain) needed to execute the fire danger model. The WXT520 sensor system can be easily deployed and installed. Other configurations of this system are designed for military usage ${ }^{4}$, and can be setup in an ad hoc way out in the field. Those kinds of weather stations may also support fire fighters in action. Then, it is necessary to have an on-the-fly integration of the attached sensors and the publication of its data in the GII to enable ubiquitous and interoperable access to the measured data from different (environmental) applications, such as the Fire Danger models.

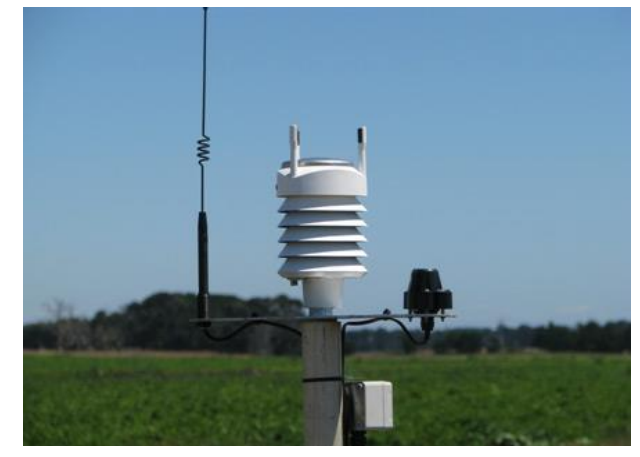

Figure 9. The Vaisala WXT520 weather station.

Before the administrator of the Vaisala WXT520 can publish its data via the GSF/SID service to a GII, a Sensor Interface Descriptor (SID) (Section 3.3) has to be created. This SID has to precisely describe the protocol of the weather station as defined in its

${ }^{4}$ http://www.vaisala.com/en/defense/products/automaticweatherstations/Pages/MAWS201M.aspx 
specification (Vaisala 2010), so that the GSF and the associated SID Interpreter can understand the incoming sensor data. Similar to other sensor systems, the WXT520 is triggered to send data via a certain command. Listing 1 shows this command and an example response of a data message that contains the latest sensor data for wind direction and speed, air temperature, relative humidity, barometric pressure, and accumulated rain (24h).

Listing 1: Example sensor command and response to retrieve data from a WXT520 station as defined in (Vaisala 2010).

Command: $\quad 0 \mathrm{R} 0<\mathrm{Cr}><\mathrm{lf}>$

Response: $\quad 0 \mathrm{RO}, \mathrm{Dx}=005 \mathrm{D}, \mathrm{Sx}=2.8 \mathrm{M}, \mathrm{Ta}=23.6 \mathrm{C}, \mathrm{Ua}=14.2 \mathrm{P}, \mathrm{Pa}=1026.6 \mathrm{H}, \mathrm{RC}=0.03 \mathrm{M}<\mathrm{Cr}>\mathrm{Cl} \mathrm{f}>$

The creation of the SID file without tool support is tedious and error-prone, since XML has to be written manually. For this reason, the visual SID Creator (Bröring et al. 2011c) enables a semi-automatic generation of SID instances. Following the wizard user interface pattern, the user of the SID Creator can define the sensor protocol, metadata, commands and processing steps in a step-by-step approach.

Figure 10 shows the part of the SID Creator that helps users to specify the structure of incoming sensor observations (raw sensor data). First, it needs to be defined how the SID interpreter retrieves data from the sensor, in this case, via the serial port. Next, the separator signs of the sensor protocol are defined. Those signs are utilized by the protocol to separate blocks within an incoming data stream, fields within those blocks and decimal numbers within fields. The screenshot shows the structure definition of incoming data from the Vaisala WXT520 weather station (Listing 1). The block definition for the air temperature values is highlighted. The end of this block is marked by a comma and the fields within the block are separated by an equal character. Two fields are defined for this block, the first one identifies the block, and the second one is the measured temperature value that is named here AirTemperature_Value for further reference.

After defining the protocol structure, the next pages of the SID Creator allow to associate defined fields with semantics. For example, the AirTemperature field can be marked as an output of the sensor. For this output, the observed property as well as the unit of measure (Section 2.2) is specified via dedicated text fields. Further the feature of interest observed by the sensor is defined by the user of the SID Creator. Those metadata are required in order to form 0\&M observations from the incoming sensor data. The metadata as well as the structure definition are stored within a SensorML file which is eventually generated by the SID Creator. 


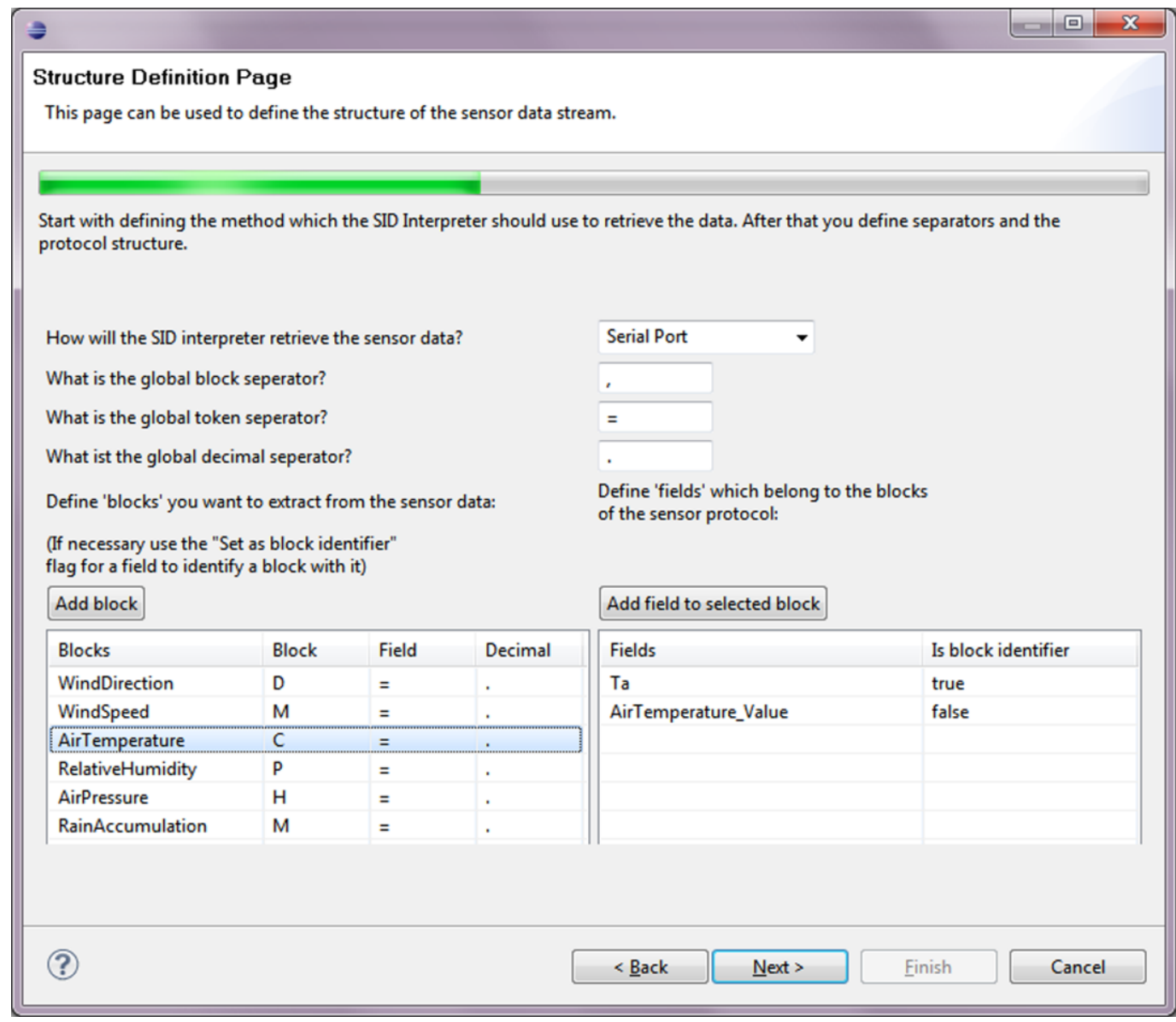

Figure 10. Structure definition page of the SID creator.

Once the SID instance is created, the next step for the sensor provider is to hand it over, together with the IP address of the sensor, to the GSF so that it can (in combination with the associated SID Interpreter) retrieve the measured data from the WXT520 to publish it in a Sensor Observation Service as part of a GII. Subsequently, the observations are available in a standardized way for an easy inclusion into environmental models. An excerpt of a WPS Execute request sent to the GSF by the sensor provider is shown in Listing 4.

Listing 4: Example of WPS Execute request to run the publication process of the GSF.

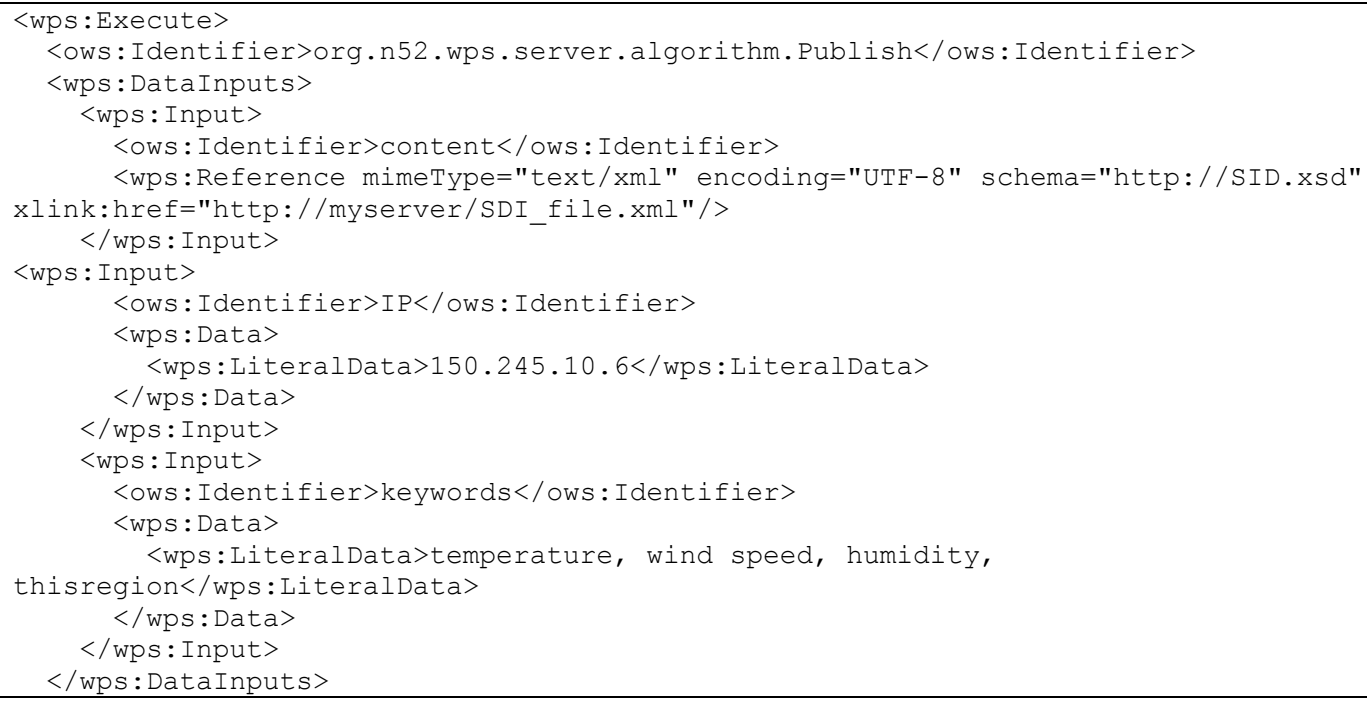


This is the request that as we can appreciate in Figure 6 is the first step that would trigger the publication process based on standard components. As previously explained this sequence ends with the publication of these observations in an standard-base Sensor Observation Service. Thus enabling the access of these observations through an interoperable service deployed in the GII to be reused as input by the Fire Danger model

\subsection{Fire Danger Model}

For this paper, we demonstrate our approach based on the use case of the EC-JRC's EFFIS and its fire danger model that produces the Fire Weather Index forecast at a pan-European scale. Currently, the Fire Danger model is run on a daily basis using the meteorological variables of temperature, relatively humidity, wind speed and daily rain. The model calculates the following six parameters, which are presented in a flow diagram in Figure 11. The specific calculations and the associated formulas of each parameter are detailed in Van Wagner (1987). A brief description of each parameter is listed as follows:

1) Fine Fuel Moisture Code (FFMC) which is the expression of the water content of litter and fine dead fuels. It provides a direct indication of the ignition and flammability of fine dead fuels;

2) Duff Moisture Code (DMC) represents the water content of a moderately thick organic layer.

3) Drought Code (DC) represents a rating of the water content of a deep, compact organic layer in the soil and provides a good indicator of seasonal drought;

4) Initial Spread Index (ISI) provides an estimate of the expected propagation of the flame front;

5) Build Up Index (BUI) represents a rating of the total fuel available for burning.

6) Fire Weather Index (FWI) represents an index of fire danger for a given area, where values range from 1.0 through 30.0 with higher values indicating higher fire severity. The calculation of the FWI can be described using the following pseudo code:

$$
\begin{aligned}
& \text { If }(B U I<80)( \\
& \quad \mathrm{f}(D M C)=0.626^{*} B U I^{0.809}+2 \\
& ) \text { else }(B U I>80)( \\
& \quad \mathrm{f}(D M C)=1000 /\left(25+\ldots 108.64 * e^{0.203 * B U I}\right) \\
& B=0.1 * I S I * \mathrm{f}(D M C) \\
& \text { If }(B>1) \\
& \quad F W I=2.72\left(0.434^{*} \log B\right)^{0.647} \\
& \text { If }(B<1) \quad F W I=\mathrm{i}
\end{aligned}
$$

Where $B$ is an intermediate form of $F W I$, and the input parameters $B U I$ and $D M C$ as defined above. 


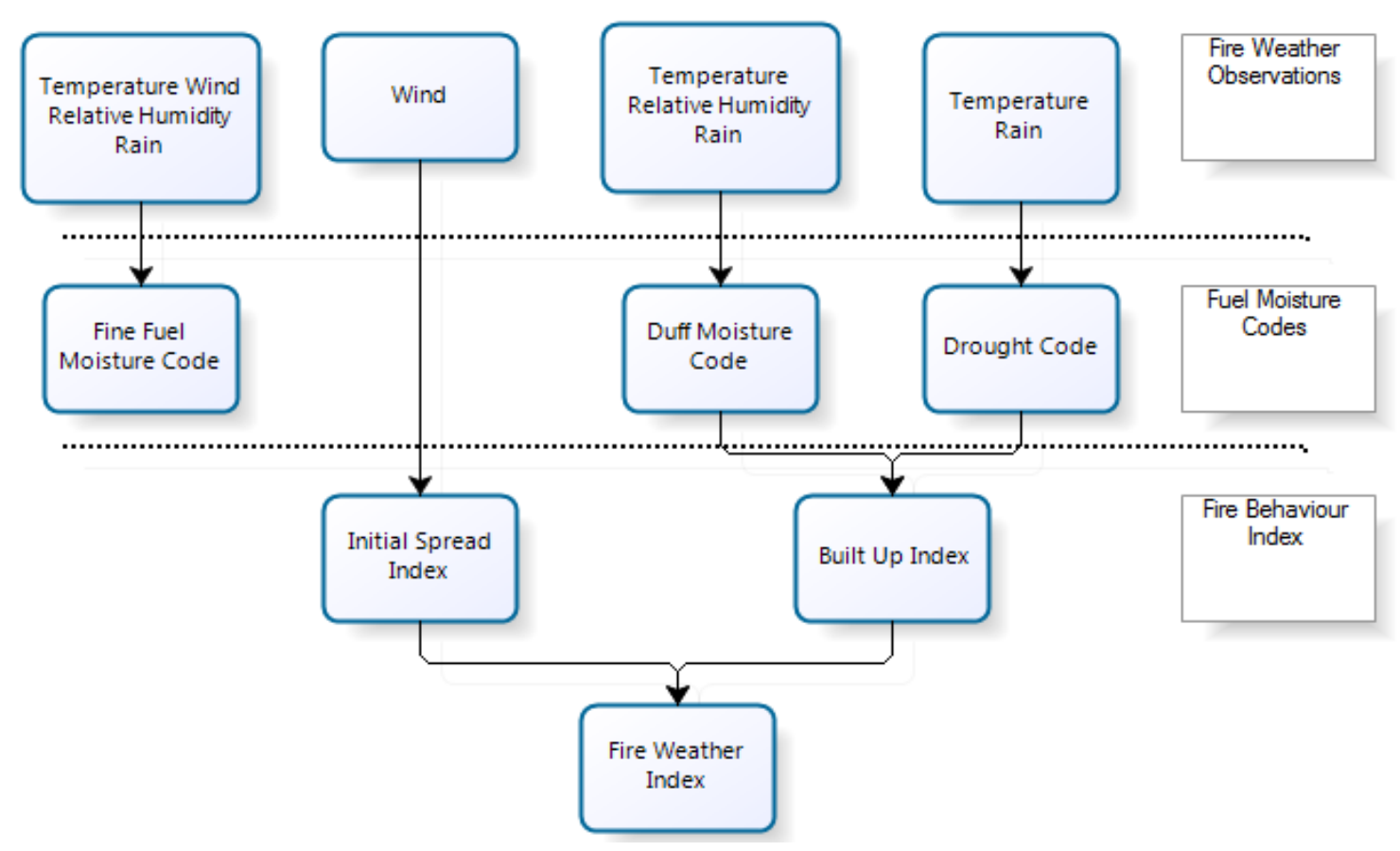

Figure 11. Schematic Structure of the FWI.

While the current FWI is produced at a pan-European level at a relatively coarse spatial resolution it becomes evident that by running the fire danger model using meteorological data retrieved from a network of locally distributed observation sensors can facilitate its production at a higher spatial resolution. These local meteorological inputs produce more localised and higher spatial resolution FWI. This would provide more detailed information to local Forest Authorities on the risks of forest fires in their locality. Based on the easy access of sensor observations it is also possible to re-run the analysis at higher temporal frequencies resulting in more up-to-date forecasts of forest fire danger. It is also conceivable that the combined use of the sensor data with topographic and local forest inventory data would allow for the development of a detailed forest fire propagation model.

The FWI published online by EFFIS (http://effis.jrc.ec.europa.eu/current-situation) is calculated on a daily basis at a spatial resolution of 1 degree (WGS84) using interpolated meteorological data from Meteo-France.

For the purposes of this study, we calculated the FWI using the meteorological observations from the 72 sensors distributed within the province of Valencia as shown in Figure 12. The observations were acquired from the $17^{\text {th }}$ of February to the $20^{\text {th }}$ of March 2012. The sensor data FWI was computed using the in-house EFFIS Fire Danger model. This ensured that we could make a direct comparison with the operationally EFFIS FWI that is calculated on a daily basis at a spatial resolution of 1 degree (WGS84) using interpolated meteorological data from Meteo France. Consequently, we extract the EFFIS FWI data for the same period as the sensor data observations and carried out the statistical comparison. 

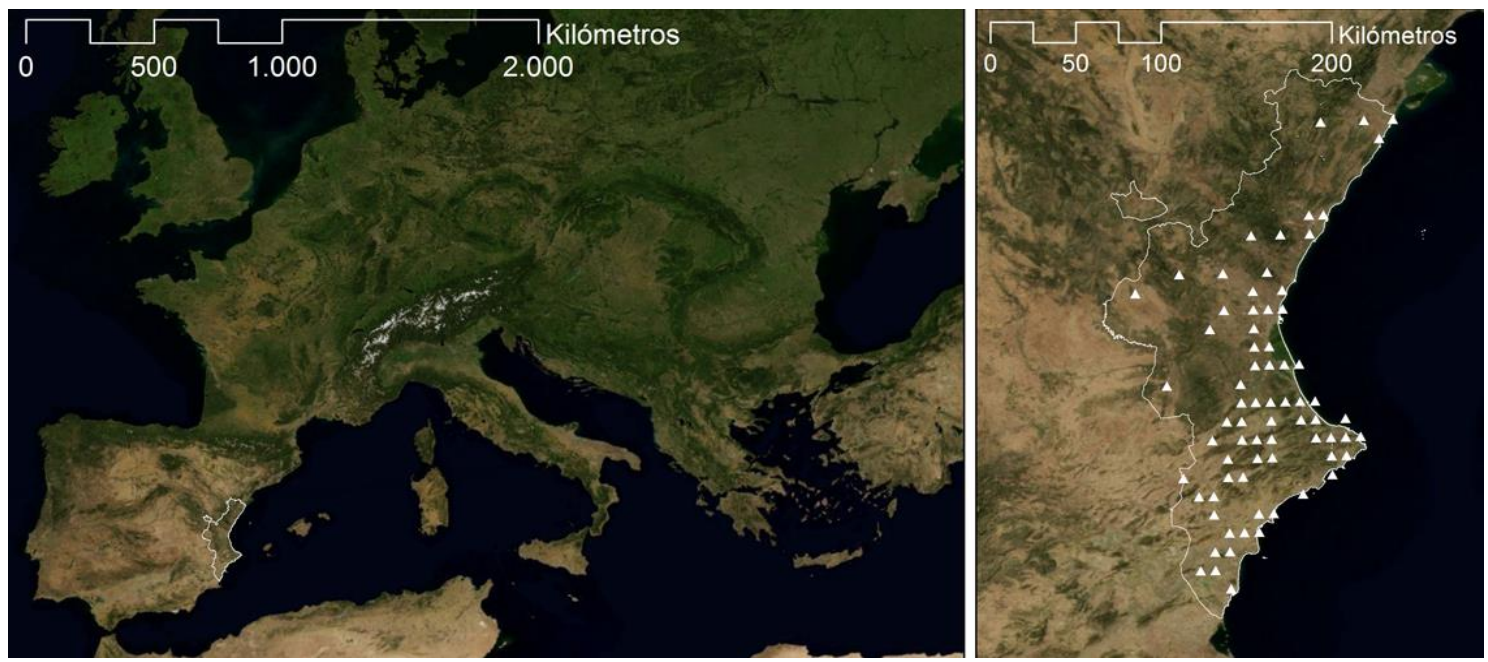

Figure 12. Detail of the region of study and the sensor network used for the use case.

\subsection{Assessment of model results impact}

Univariate statistics for the FWI and FFMC were computed from the Meteo France as well as the sensor data for the period from the $1^{\text {st }}$ till the $20^{\text {th }}$ of March 2012 (Table 1). Furthermore, average estimates of FWI were computed for all observations within the study area for both datasets. This provided an insight into the trends of the model outputs.

The following results compare the differences between the FWI and the FFMC for the province of Valencia based on the Meteo France and Sensor Data provided by SOS. Univariate statistics are computed for the period from the $1^{\text {st }}$ March until the $20^{\text {th }}$ March 2012. Although meteorological observations were available for a longer period $\left(17^{\text {th }}\right.$ February - $20^{\text {th }}$ March), it was enough to calibrate the model using a period of approximately two weeks to obtain stable observations.

Table 1 Univariate Statistics FWI / FFMC from Meteo France \& sensor data; 1st - 20th March 2012

\begin{tabular}{|l|l|l|l|l|}
\hline Variable & Minimum & Median & Mean & Maximum \\
\hline FWI (Meteo France) & 1.8 & 10.8 & 10.34 & 21.8 \\
\hline FWI (Sensor Data) & 1.17 & 6.78 & 6.612 & 9.3 \\
\hline FFMC (Meteo France) & 61.59 & 87.63 & 84.7 & 89.42 \\
\hline FFMC (Sensor Data) & 40.7 & 90.1 & 87.05 & 91.58 \\
\hline
\end{tabular}

Figures 13 \& 14 show the Sensor Data FWI (SD_FWI) and Meteo France FWI (MF_FWI). Although, the SD_FWI is lower than for the Meteo France data, both FWIs display a common trend. The graphs indicate that from the $10^{\text {th }}$ of March onwards, both FWI follow a similar trend, which would indicate that the model outputs are becoming more stable. 


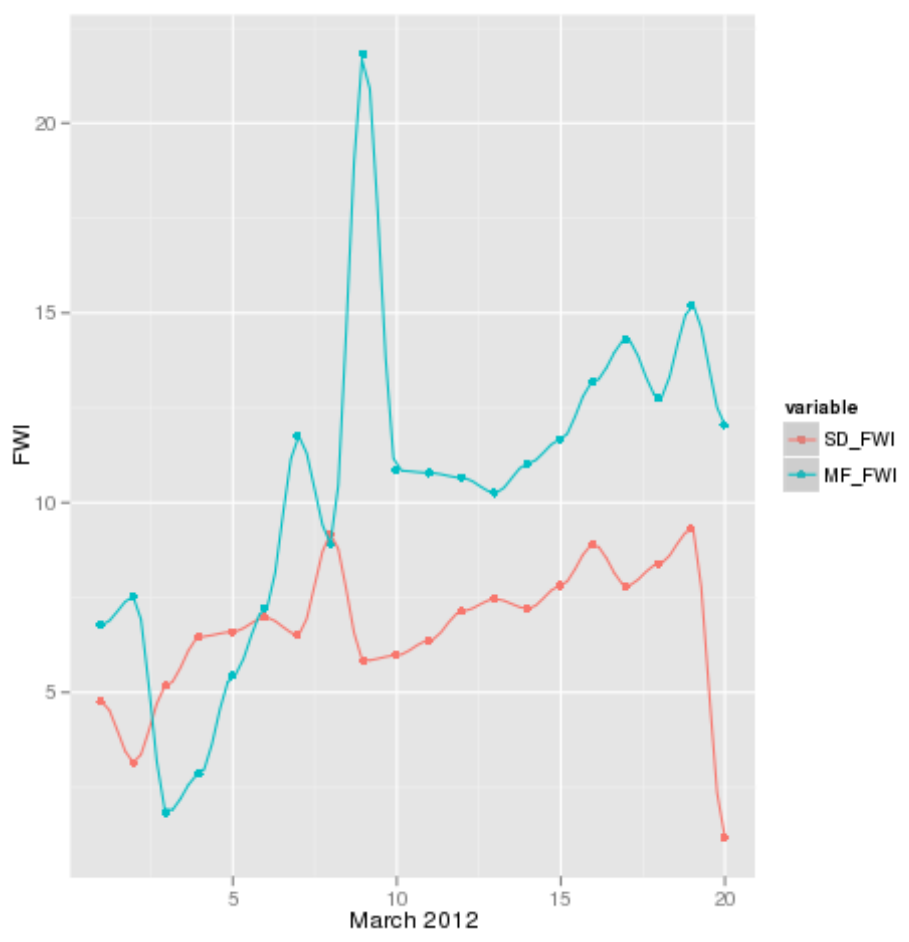

Figure 13. FWI estimates from Sensor Data (SD_FWI) and Meteo France (MF_FWI) data for the period 1st March - 20th March 2012.

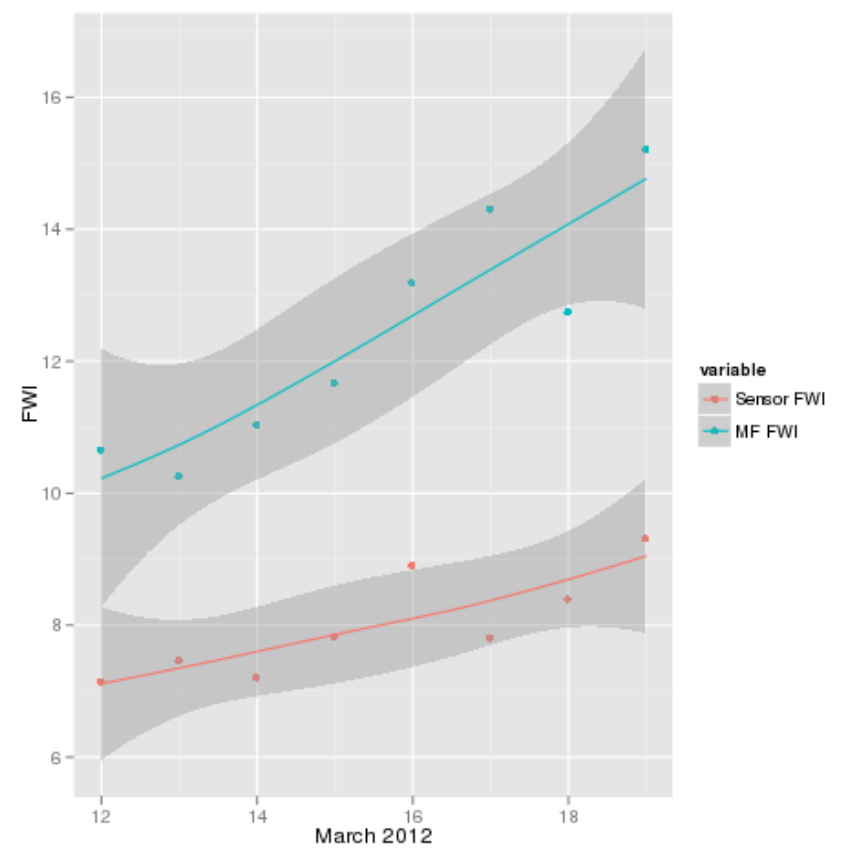

Figure 14. FWI estimates from Sensor Data and Meteo France data for the period 12th March - 20th March 2012.

On the other hand, the FFMC derived from the sensor data (Figure 15) is slightly higher, but is closer to the estimate for the Meteo France data. However, the $3^{\text {rd }}$ of March appears to be an outlier for the Meteo France data, whilst another outlier is detected on the $20^{\text {th }}$ of March for the Sensor data. 


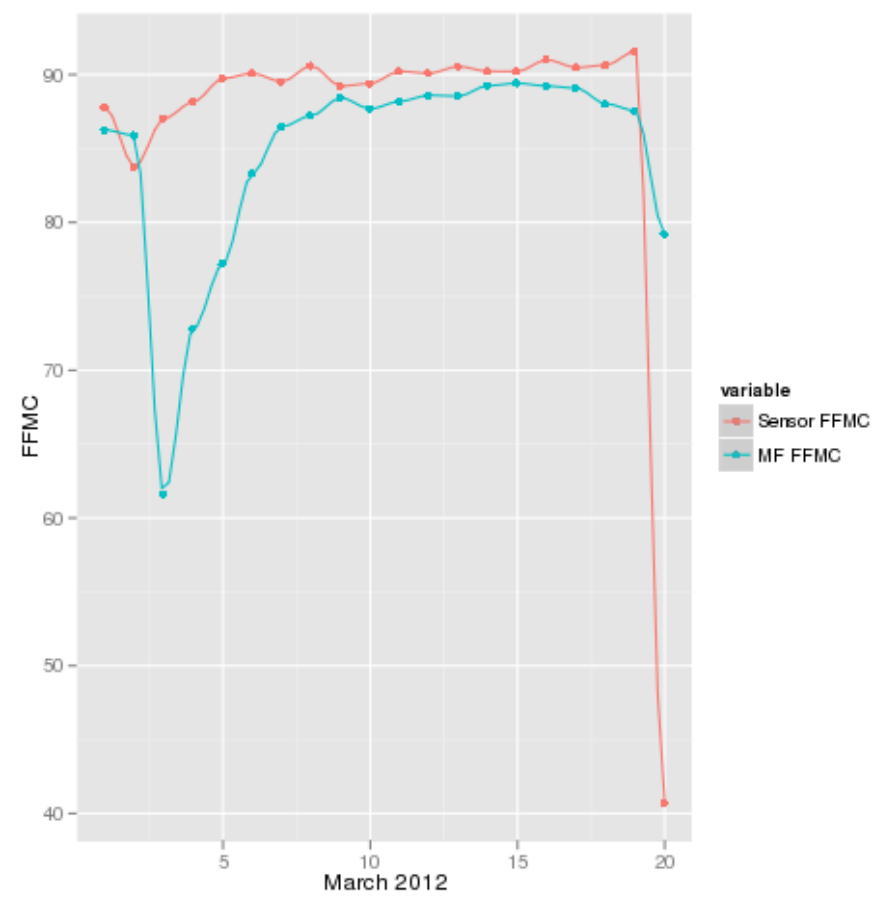

Figure 15. FFMC estimates from sensor data and Meteo France data for the period 1st March - 20th March 2012.

Figure 16 shows the spatial distribution of these results for the FWI on the $18^{\text {th }}$ of March, 2012 for the province of Valencia. Based on the EFFIS for Meteo France data, the FWI is interpolated at pan-European scale. Within this study, we demonstrate that the FWI is an over-estimate due to the coarse resolution of the meteorological observations. The map on the right represents the fire danger model results using sensor data from the Valencia region on the same date. The FWI was calculated for each sensor position and subsequently the surface was created by running an Inverse Distance Weighting interpolation using 12 points. In order to be able to compare both datasets, we have made a five classes classification of equal size ranging a FWI between 0 and 25 . 

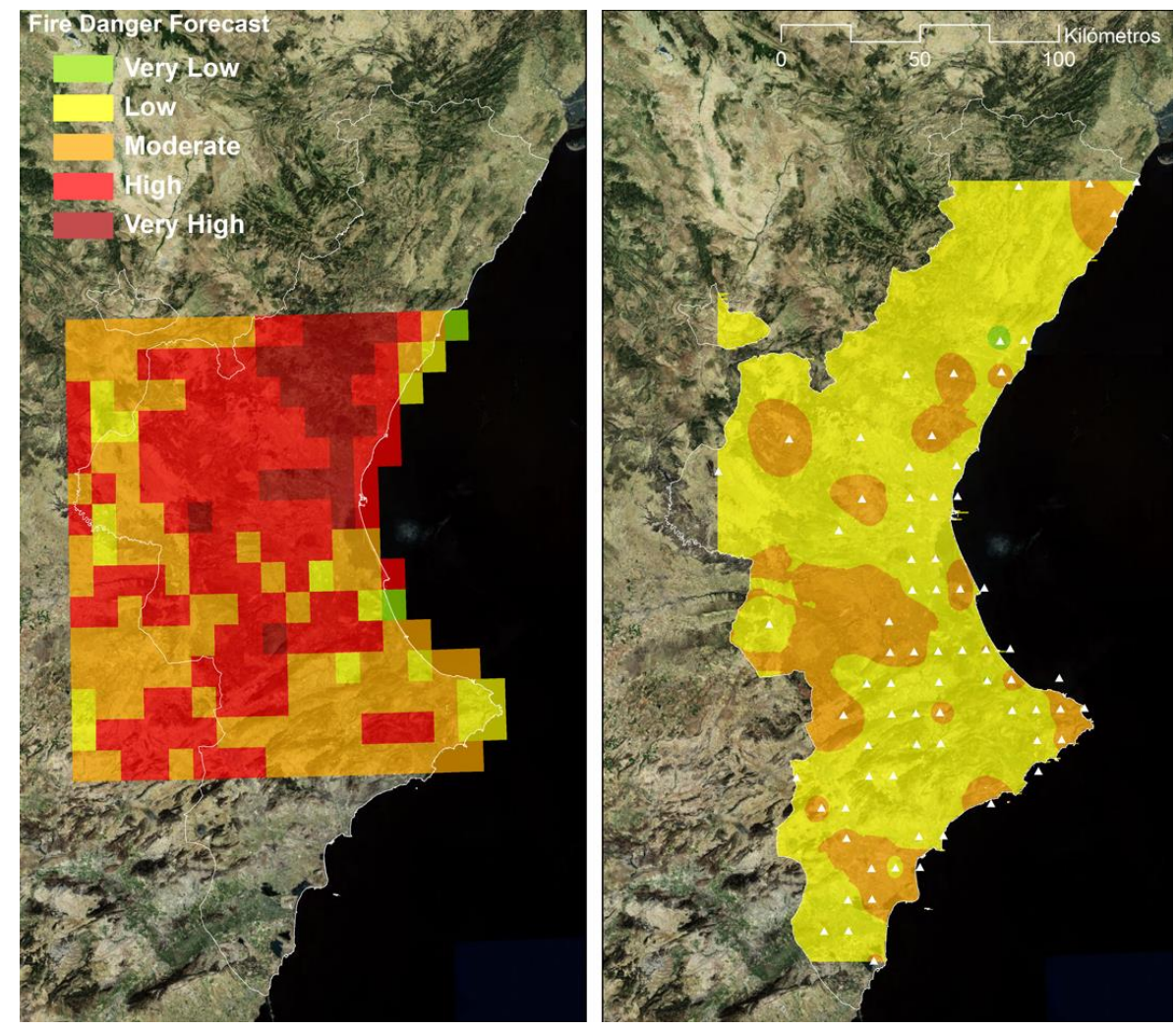

Figure 16. left) FWI run with the Meteo France data; right) FWI results when using sensor data fromValencia region, both on 18th of March, 2012

\section{Conclusions and Future Work}

Environmental monitoring such as fire danger assessment depends on the availability of high spatio-temporal resolution data to provide forecast information on a local scale, which can consequently enable the better management of forest and wildland fire events. In particular, meteorological data provided by in-situ sensors is an essential input for fire danger models. Therefore, the required geospatial data in general and sensor observations need to be available in an interoperable manner through GII. We face two key challenges to increase the availability of this data in GII. Firstly, the variety of sensor protocols defined by manufacturers turns the integration of such data into an arduous task. Secondly, GII, which are traditionally top-down infrastructures do not provide the users with interoperable mechanisms to publish data. Instead, publishing any kind of content in GIIs requires the knowledge about a variety of standards and their implementations.

In this article, we addressed these two challenges. We described a novel approach for automating the integration and publication of in-situ sensor data wrapped via a standardized service interface. We have combined the GEOSS Service Factory (GSF) and the Sensor Interface Descriptors (SID), which together deployed in a GII, provide an approach to firstly interpret data from various sensors and secondly publish those data as observations in an interoperable manner. This approach supports sensor providers in publishing their measured data in GII.

The GSF is responsible for publishing incoming data as GII services and has itself the standardized interface of a Web Processing Service (WPS). A sensor provider can use that WPS interface to send the standard Execute request to the GSF which contains the address of a sensor as well as an SID file. This SID file describes the sensor protocol in detail so that it enables the SID interpreter, associated with the GSF, to understand and transform the 
incoming sensor data into the standardized O\&M format. Then, the GSF component uploads the generated O\&M observations to a Sensor Observation Service (SOS). Henceforth, the observations are available to clients via the standardized interface of the SOS.

For sensor network providers it would be beneficial to utilize SID and GSF mechanisms to make sensors and sensor data be available via GII. In particular, it is beneficial, when SIDs exist for their utilized sensors since an SID acts as a well-defined description of the sensor protocol. An SID Interpreter can make use of the knowledge contained within the SID to transform incoming sensor data streams to standardized data formats. Also, in case of this work the SID produced for integrating the Vaisala weather station can, in the future, be reused by others and in different contexts. Previous work has demonstrated the generation of SIDs for various kinds of sensors (e.g., water level, salinity, or temperature) which can already be reused (Bröring 2012a).

However, there is a tremendous number of manufacturer specific sensor protocols (Bröring et al. 2010a), and consequently the SID interpreter can only support a limited set of those protocols. Plain ASCII text encodings which use dedicated separator signs to structure the sensor data streams (e.g. the marine sensor instrument Seabird SBE 37; SeaBird-Electronics 2010) are supported. Also, binary encoded protocols with fixed width data block structures (e.g. the protocol HOBI Labs HydroScat oceanographic fluorometer; HOBILabs 2008) can be described. Currently not covered by the SID specification are for example ASCII text protocols which define the structure of their data blocks by giving a fixed-width for the data block tokens. A future line of our research will extend the SID specification as well as our SID interpreter to overcome the current limitations in order to support a broader variety of sensor protocols (Bröring 2012a).

To increase sensor observation discovery, our approach generates a minimum set of metadata records that is published in a Catalog. In future work, more detailed metadata could be generated. Current version only generates discovery-purpose metadata elements, however other metadata such as quality information or other sensor-related data in order to be able to calibrate observations before feeding models are also necessary. Among others, this line of research would include the integration of automatic metadata generation packages (Abugessaisa, 2010). Moreover, for sensor data discovery the Sensor Instance Registry (SIR) (Jirka et al, 2009) is a proposal currently being discussed at the OGC which API is feasible to be used, by the Discovery Factory, for registering sensors due to the generic design of the GSF.

Increasing the interoperable availability of in-situ sensor data provides scientific models, e.g. to assess fire danger risks, with data to improve their accuracy. Currently, many Fire Danger Indices, including the Fire Weather Index are calculated on a daily basis using continental scale meteorological data. The coarse nature of these meteorological observations means that they can only be used to calculate a Fire Weather Index at a relatively low spatial resolution. In the case of the FWI, it is calculated for a grid with a spatial resolution that ranges from 36 to $45 \mathrm{~km}$. While this is sufficient at a pan-European scale, it is clear that it would potentially miss local changes or anomalies in weather conditions caused by topography or other conditions. The research presented in this paper demonstrates that it is feasible to use sensor data to compute an FWI at a higher spatial resolution (e.g. in the range of $5-10 \mathrm{~km}$ ), but with a more limited geographic extent, for instance at a local or regional scale. This forecast information could be better integrated into local or regional forest fire monitoring and management plans.

The comparison of the FWI and FFMC from the Sensor Data and Meteo France provided promising results. Despite the limited amount of Sensor Data observations, the study demonstrated that it is possible to run the EFFIS Fire Danger model using meteorological data from a dense network of Sensors. It also showed that good results were achieved for 
the period from the $1^{\text {st }}$ of March until the $20^{\text {th }}$ of March and the estimates derived from the Sensor data observed the same general trend as the estimates for Meteo France data. The lower sensor data FWI can be explained by the fact that the input meteorological data are different from the interpolated Meteo France data and it is conceivable that the estimates are more accurate for the localised area.

This work has provided a good demonstration and positive results to start producing a more localised FWI on an operational basis. Clearly, a longer term analysis of the sensor data FWI with respect to the Meteo France FWI would enable a deeper insight into the model performance and the overall trend.

We have presented an approach that lowers the barrier to deal with complex data formats, and standard service interfaces which are used in GIIs to increase interoperability and interlinking, however recent research lines (e.g., Granell et al, 2013, Mazzetti et al. 2009) point out the necessity to improve this interlinking by establishing resource-oriented architectures following REST principles. We did not address this here but it is a possible future research topic, to investigate how more light-weight architectures may facilitate the interaction with resources (data and models).

The use-case scenario presented within this paper describes how the accuracy of the results of a forest fire danger model can be improved by our approach that increases sensor observations related to meteorological data. The presented approach and the proposed technology and infrastructure, based on standard services and data formats, could be reused to monitor other natural hazards, such as flooding, flash floods, or at a longer temporal scale to monitor climate change, such as the extent of drought.

\section{Acknowledgements}

This work has been financially supported by the European FP7 Project nr. 226487 called EuroGEOSS, the project Flexible and Efficient Integration of Sensors and Sensor Web Services funded by the ERDF program for NRW (contract number N 114/2008), as well as the $52^{\circ}$ North Initiative for Geospatial Open Source Software (http://52north.org), and by the post-doc scholarship of the Institute for Geoinformatics, University of Münster. Many thanks to Vaisala Oyi, Helsinki, Finland (http://www.vaisala.com) for providing the weather station sensor system used in the case study.

\section{Bibliography}

Aberer, K., Hauswirth, M., Salehi, A. (2006). A Middleware for Fast and Flexible Sensor Network Deployment. In: Dayal, U.; Whang, K.-Y.; Lomet, D. B.; Alonso, G.; Lohman, G. M.; Kersten, M. L.; Cha, S. K. \& Kim, Y.-K. (ed.): 32nd International Conference on Very Large Data Bases, ACM, 1199-1202.

Abugessaisa, I. (2010). Geospatial metadata extraction from product description document applying methods from ontology engineering. Int. J. Metadata, Semantics and Ontologies, Vol. 5, No. 4, pp. 321-332.

Arroyo, L., Pascual, C., Manzanera, J. (2008) Fire models and methods to map fuel types: The role of remote sensing. Forest Ecology and Management 256 pp.1239 -- 1252

Botts, M., Percivall, G., Reed, C., \& Davidson, J. (2008). OGC Sensor Web Enablement: Overview And High Level Architecture. GeoSensor Networks, Lecture Notes in Computer Science (pp. 175-190). Heidelberg: Springer. 
Botts, M., \& Robin, A. (2007). Sensor Model Language (Implementation specification No. 07-000). Wayland, MA, USA: OGC. Retrieved from http://www.opengeospatial.org/standards/sensorML

Bröring, A. (2012a). Automated Onthefly Integration of Geosensors with the Sensor Web. ITC dissertation, 211. Enschede, The Netherlands

Bröring, A., Stasch, C. \& Echterhoff, J (eds), (2012). Sensor Observation Service Interface Standard 2.0 (Implementation specification No. OGC 12-006). Wayland, MA, USA: OGC. Retrieved from https://portal.opengeospatial.org/files/?artifact_id=47599

Bröring, A., Echterhoff, J., Jirka, S., Simonis, I., Everding, T., Stasch, C., Liang, S. \& Lemmens, R. (2011a). New Generation Sensor Web Enablement. Sensors, 11(3), pp. 2652-2699.

Bröring, A., F. Bache, T. Bartoschek \& C.P.J.M. van Elzakker (2011c): The SID Creator: A Visual Approach for Integrating Sensors with the Sensor Web. In: S. Geertmann, W. Reinhardt \& F. Toppen (Eds.), Advancing Geoinformation Science for a Changing World, The 14th AGILE International Conference on Geographic Information Science. 18.-21. April 2011. Utrecht, Netherlands. Lecture Notes in Geoinformation and Cartography, Springer, Volume 1, pp 143-162.

Bröring, A., Maué, P., Janowicz, K., Nüst, D. \& Malewski, C. (2011d): Semantically-Enabled Sensor Plug \& Play for the Sensor Web. Sensors, 11(8), pp. 7568-7605.

Bröring, A., S. Below \& T. Foerster (2010a): Declarative Sensor Interface Descriptors for the Sensor Web. In: Brovelli, M., Dragicevic, S., Li, S. \& Veenendaal, B. (Eds.), WebMGS 2010: 1st International Workshop on Pervasive Web Mapping, Geoprocessing and Services. 26.-27. August 2010. Como, Italy. ISPRS, 2010, Volume 38.

Camia, A., Bovio, G. (2000) European Forest Fire Information System (EFFIS) Meteorological Indices.

Camia, A., Barbosa, P., Amatulli, G., San-Miguel Ayanz, J. (2006) Fire danger rating in the European Forest Fire Information System (EFFIS): current developments. 5th International Conference on Forest Fire Research, Coimbra, Portugal, 27th-30th November 2006.

Camia, A., Amatulli, G. (2010). Climatology of FWI over Europe: fire danger anomalies and index percentile rankings. In: Viegas D.X. (Ed.) Proceedings of the VI International Conference on Forest Fire Research, ADAI/CEIF, Coimbra, Portugal. Novemeber 2010.

Christian, E. (2005) Planning for the Global Earth Observation System of Systems (GEOSS) Space Policy 21 pp.105-109.

Cox, S. (2007). Observations and Measurements - Part 1 - Observation schema (Implementation specification No. 07-022r1). Wayland, MA, USA: OGC. Retrieved from http://www.opengeospatial.org/standards/om

Conover, H., Berthiau, G., Botts, M., Goodman, H.M., Li, X., Lu, Y., Maskey, M., Regner, K., Zavodsky, B., 2010. Using sensor web protocols for environmental data acquisition and management. Ecological Informatics 5 (1), 32-41

Craglia, M., Goodchild, M.F., Annoni, A., Câmara, G., Gould, M., Kuhn, W., Mark, D., Masser, I., Maguire, D., Liang, S., Parsons, E. (2008). Next-Generation Digital Earth: A position paper from the Vespucci Initiative for the Advancement of Geographic Information Science. International Journal of Spatial Data Infrastructures Research 3, 146-167. 
Díaz, L, Granell, C., Gould, M., Huerta, J. (2011). Managing user generated information in geospatial cyberinfrastructures. Future Generation Computer Systems 27 (3), 304-314

Díaz, L., Shade, S. (2011) GEOSS Service Factory: Assisted Publication of Geospatial Content. In S. Geertman, W. Reinhardt, F. Toppen (Eds): Advancing Geoinformation Science for a Changing World (Proceedings of the 14th AGILE Conference), Springer, LNGC, pp. 423-442

Gershenfeld, N., Krikorian, R., Cohen, D. (2004). The Internet of Things. Scientific American, 291(4):76-81, 2004.

Gigan, G. \& Atkinson, I. (2007). Sensor Abstraction Layer: A Unique Software Interface to Effectively Manage Sensor Networks. 3rd International Conference on Intelligent Sensors, Sensor Networks and Information (ISSNIP 2007), IEEE, pp. 479-484

Granell, C., Díaz, L., Schade, L., Ostländer, N., Huerta, J. (2013) Enhancing Integrated Environmental Modelling by Designing Resource-Oriented Interfaces. Environmental Modelling \& Software 39 pp. 229-246

Granell, C., Díaz, L., Gould, M. (2010) Service-oriented applications for environmental models: Reusable geospatial services. Environmental Modelling \& Software 25 pp. 182198

Foerster, T., Schaeffer, B., Baranski, B., \& Brauner, J. (2011). Geospatial Web Services for Distributed Processing - Applications and Scenarios. In P. Zhao \& L. Di (Eds.), Geospatial Web Services: Advances in Information Interoperability (pp. 245-286). Hershey, PA: IGI Global.

Fook, K.D., Monteiro, A.M.V., Câmara, G., Casanova, M.A., Amaral, S., 2009. Geoweb Services for Sharing Modelling Results in Biodiversity Networks. Transactions in GIS 13 (4), 379399

Havlik, D., Schade, S., Sabeur, Z., Mazzetti, P., Watson, K., Berre, A., Mon, J., (2011). From Sensor to Observation Web with Environmental Enablers in the Future Internet. Sensors $11,3874-3907$

Heuvelink, G. (1998) Error Propagation in Environmental Modeling with GIS, Taylor \& Francis. Location?

HOBILabs (2008). HydroScat-4 Spectral Backscattering Sensor - User's Manual. Online Available: http://purl.oclc.org/sensor/HOBILabs/Hydroscat4

Hui, J. W. \& Culler, D. E. (2008). IP is Dead, Long Live IP for Wireless Sensor Networks. In SenSys 2008: Proceedings of the 6th ACM Conference on Embedded Network Sensor Systems, pp 15-28, New York, NY, USA, 2008. ACM.

Jirka, S., Bröring, A \& Stasch, C. (2009). Discovery Mechanisms for the Sensor Web. Sensors, 9(4), pp. 2661-2681.

ISO 19115. (2003). Geographic information Metadata. Available at: http://www.iso.org/iso/catalogue_detail.htm?csnumber=26020

Klopfer, M. \& Simonis, I. (2009). SANY - An Open Service Architecture for Sensor Networks. SANY Consortium.

Lautenbacher, C. (2006). The Global Earth Observation System of Systems: Science Serving Society. Space Policy, 22, 8-11. 
Lee, B.S, Alexander, M.E., Hawkes, B.C., Lynham, T.J., Stocks, B.J., Englefield, P. (2002) Information systems in support of wildland fire management decision making in Canada. Computers and Electronics in Agriculture 37:185-198

Lee, C., Percivall, G. (2008). Standards-Based Computing Capabilities for Distributed Geospatial Applications. Computer 41(11): 50-57.

Masser, I., 2005. GIS Worlds: Creating Spatial Data Infrastructures. ESRI Press, Redlands

Mazzetti, P., Nativi, S., Caron, J. (2009) RESTful Implementation of Geospatial Services for Earth and Space Science Applications. International Journal of Digital Earth 2(1): 40-61. McArthur, A.G. (1966) Weather and grassland fire behaviour. Australian Forestry and Timber Bureau Leaflet, no. 107

Mykkänen, J., Tuomainen, M. (2008). An evaluation and selection framework forinteroperability standards. Information and Software Technology, vol 50, issue 3, pp. 176-197.

Na, A., and Priest, M. (eds), 2007. Sensor Observation Service (Implementation specification No. 06-009r6). Wayland, MA, USA: OGC. Retrieved from http://portal.opengeospatial.org/files/?artifact_id=26667

Nebert, D. (ed), 2004. Developing Spatial Data Infrastructures: The SDI Cookbook v.2.0. Global Spatial Data Infrastructure (GSDI), http://www.gsdi.org

Nebert, D., Whiteside, A. 2004. OGC-Catalogue Services specification (version: 2.0). OpenGIS Project Document 04-021r2, Open GIS Consortium.

Nittel, S., Labrinidis, A. and Stefanidis, A. (2008): Introduction to advances in geosensor networks. Lecture Notes in Computer Science 4540, pp. 1-6.

Percival, G. (ed). 2008. OGC Reference Model. Open Geospatial Consortium Inc. Date: 200811-11. Reference number: OGC 08-062r4. Version: 2.0

Rothermel, R. C. (1972) A mathematical model for predicting fire spread in wildland fuelds. Report. No. RP INT-115. SDA Forest Service, Ogden, UT.

Rizzoli, A.E., Leavesley, G., Ascough, J.C., Argent, R.M., Athanasiadis, I.N., Brilhante, V., Claeys, F.H.A., David, O., Donatelli, M., Gijsbers, P., Havlik, D., Kassahun, A., Krause, P., Quinn, N.W.T., Scholten, H., Sojda, R.S., Villa, F., 2008. Integrated Modelling Frameworks for Environmental Assessment and Decision Support. In: A.J. Jakeman et al. (edi): Developments in Integrated Environmental Assessment, pp 101-118

Schade, S., Díaz, L. (2010) Supporting Content Provision in Environmental Information Infrastructures. Environmental Information Systems and Services: Infrastructures and Platforms Workshop (Workshop at EnviroInfo2010). Bonn/Cologne, Germany, October 2010.

Schut, P. (ed). 2007. OGC Web Processing Service (WPS) version 1.0.0. OGC Standard Document, Open Geospatial Consortium.

Sea-Bird-Electronics (2010). SBE 37-SMP MicroCAT - Conductivity and Temperature Recorder with RS-232 Interface and Integral Pump. Online Available: http://purl.oclc.org/sensor/Seabird37MP 
Šerić, L., Stipaničev, D., Štula, M. (2011). Observer network and forest fire detection. Information Fusion. 12 (3), pp. 160-175.

Stipaničev, D., Bodrožić, Lj., Štula, M., Environmental (2007). Intelligence based on Advanced Sensor Networks, Proc.of 14th Int.Conference onSystems, Signals and Image Processing, Maribor, Slovenija, pp. 27-30.

Vaisala (2010). User's Guide - Vaisala Weather Transmitter WXT520. Vaisala Oyi. Helsinki, Finland. Online Available: http://www.vaisala.com/Vaisala\%20Documents/User\%20Guides\%20and\%20Quick\%20Ref\%20Guides/WXT520_User_Guide_in_English.p df

Van Wagner,C.E. and Pickett, T.L. (1987) Equation and Fortran program for the Canadian Forest Fire Weather Index System. Canadian Forestry Service, Forestry Technical Report 33. Ottawa, Canada.

Van Wagner, C.E. (1987) Development and Structure of the Canadian Forest Fire Weather Index System. Canadian Forestry Service, Technical Report 35, pp. 37. Ottawa Canada.

Vasconcelos, M., Guertin, D.P. (1992) FIREMAP - Simulation of Fire Growth with a Geographic Information System. International Journal of Wildland Fire 2(2) pp.87-96.

Walter, K. \& Nash, E. (2009). Coupling Wireless Sensor Networks and the Sensor Observation Service - Bridging the Interoperability Gap. 12th AGILE International Conference on Geographic Information Science 2009, Online Available: http://plone.itc.nl/agileold/Conference/2009-hannover/shortpaper.htm 